\title{
Structural determinants of calcium binding beyond the EF-hand binding site: a study of alpha parvalbumins
}

Kalyan Immadisetty*, Bin Sun, and Peter Kekenes-Huskey

September 18, 2020 


\section{Abstract}

Parvalbumin (PV) is a calcium binding protein expressed in humans, fish and avian species. In these organisms, the calcium $\left(\mathrm{Ca}^{2+}\right)$ affinities of specific PV isoforms can vary by orders of magnitude. Despite the availability of high resolution structural data for many PV isoforms, the structural bases for how such proteins confer widely-varying divalent $\mathrm{Ca}^{2+}$ affinities and selectivities against common ions like magnesium $\left(\mathrm{Mg}^{2+}\right)$ has been difficult to rationalize. We therefore conducted molecular simulations of several $\alpha$-pavalbumin $(\alpha$-parvalbumin $(\alpha \mathrm{PV}))$ constructs with $\mathrm{Ca}^{2+}$ affinities in the micromolar to nanomolar ranges to identify properties of conformations that contribute to their wide-ranging binding constants and selectivities against $\mathrm{Mg}^{2+}$. Specifically, we examined a D94S/G98E construct with a reported lower $\mathrm{Ca}^{2+}$ affinity $(\approx-18.2$ $\mathrm{kcal} / \mathrm{mol})$ relative to the WT $(\approx-22 \mathrm{kcal} / \mathrm{mol})$, an S55D/E59D variant with enhanced affinity $(\approx-24$ $\mathrm{kcal} / \mathrm{mol})$, and a truncated variant of $\alpha \mathrm{PV}$ with weak affinity $(\approx-12.6 \mathrm{kcal} / \mathrm{mol})$. We performed molecular dynamics simulations of these constructs and assessed their $\mathrm{Ca}^{2+}$ and $\mathrm{Mg}^{2+}$ binding properties using scores from molecular mechanics generalized Born approximation (MM/GBSA), ion/oxygen coordination patterns and thermodynamics via mean spherical approximation (MSA) theory, as well as via metrics of protein structure and hydration. Our key findings are that although MM/GBSA and MSA scores successfully rank-ordered the variants according to their previously-published affinities and $\mathrm{Mg}^{2+}$ selectivity, importantly, properties of $\mathrm{Ca}^{2+}$ loops in CBPs such as coordination, and charge are alone insufficient to rationalize their binding properties. Rather, $\mathrm{Ca}^{2+}$ affinity and selectivity against $\mathrm{Mg}^{2+}$ are emergent properties stemming from both local effects within the proteins' ion binding sites as well as non-local contributions from protein folding and solubility. Our findings broaden our understanding of the molecular bases governing $\alpha \mathrm{PV}$ ion binding that are likely shared by many $\mathrm{Ca}^{2+}$ binding proteins.

\section{Introduction}

Parvalbumin (PV) comprise a family of proteins that shape $\mathrm{Ca}^{2+}$ signaling in many physiological processes, including neural function, contractility, and immune responses [1]. Given the breadth of PV isoforms found in nature with dramatically different $\mathrm{Ca}^{2+}$ and $\mathrm{Mg}^{2+}$ affinities [2, 3, 4, 5], these proteins have been popular targets for biochemical and structural characterizations to elucidate the molecular basis of $\mathrm{Ca}^{2+}$-affinity $[6,7,8,9,10,11]$, or as gene therapies for diseases involving calcium dysregulation $[12,13]$. Alpha PV $(\alpha \mathrm{PV})$ is a member of the PV family and is expressed in fast twitch muscle fibers, GABA-ergic neurons and the organ of Corti [14]. $\alpha \mathrm{PV}$ is unique in its extraordinarily high $\mathrm{Ca}^{2+}$ affinity relative to other $\mathrm{PV}$ isoforms and selectively binds $\mathrm{Ca}^{2+}$ over $\mathrm{Mg}^{2+}$ by $\approx 10 \mathrm{kcal} / \mathrm{mol}[15,16]$. $\alpha \mathrm{PV}$ has also been a target for detailed mutagenesis studies $[17,16,15]$ to rationalize its $\mathrm{Ca}^{2+}$ binding properties including site-directed mutations found to reduce (D94S/G98E $\alpha \mathrm{PV}$ ) or further increase (S55D/E59D $\alpha \mathrm{PV}$ ) [15] the intrinsic $\mathrm{Ca}^{2+}$ affinity of $\alpha \mathrm{PV}$, as well as a truncated construct $(\Delta \alpha \mathrm{PV})$ that exhibited dramatically reduced $\mathrm{Ca}^{2+}$ binding [18].

Many Ca-binding protein (CBP)s including $\alpha \mathrm{PV}$ contain helix-loop-helix 'EF-hand' metal-binding motifs 
that have evolved too strongly and selectively bind $\mathrm{Ca}^{2+}$. EF hands have a rich evolutionary history that reflects a high degree of sequence homology across protein types, isoforms and species [19]. Ca ${ }^{2+}$ affinity in CBPs such as $\alpha \mathrm{PV}$ has been shown to be strongly dependent on an EF-hand's sequence identity, binding site loop length and tertiary structure (reviewed in [20, 21]). Further, selective binding of Ca ${ }^{2+}$ over other mono- and divalent ions, including $\mathrm{Mg}^{2+}$, are believed to leverage structural properties of the $\mathrm{Ca}^{2+}$ binding loops in EF hands, such as the placement of ion-coordinating polar ligands that exploit the unique size and charge density of $\mathrm{Ca}^{2+}[21,22]$. Beyond the $\mathrm{Ca}^{2+}$ binding loops, myriad structural and dynamic factors tune the affinity of $\mathrm{Ca}^{2+}$ binding, including the packing of EF-hand helices, the thermodynamics of exchanging coordinated solvent with metal ion, modulation of secondary structural elements, and structural coupling between adjacent EF-hands [20]. However, the degree to which these factors contribute to the high $\mathrm{Ca}^{2+}$ affinities exhibited have not been systematically examined in $\alpha \mathrm{PV}$. Insights into these factors could not only inform upon PV proteins' functions in buffering intracellular $\mathrm{Ca}^{2+}$ levels [15], but could extend our understanding of $\mathrm{Ca}^{2+}$ sensing in the broad CBP family.

Predicting the energetics of $\mathrm{Ca}^{2+}$ and $\mathrm{Mg}^{2+}$ binding and structural changes that follow could give important insights into PV proteins, but has remained challenging. Bioinformatics techniques have made strides in identifying binding domains in $\mathrm{Ca}^{2+}$ binding proteins based on protein sequences [23, 24, 23]. Other algorithms that account for the geometric configuration of $\mathrm{Ca}^{2+}$ chelating ligands to achieve optimal coordination geometries have further improved EF-hand prediction [25] and have helped to rationalize selectivity against other ions like $\mathrm{Mg}^{2+}$ [26]. Molecular dynamics (MD) simulations [27] have facilitated detailed analyses of $\mathrm{Ca}^{2+}$ binding in intact proteins, using force fields fit to quantum chemical calculations of metal oxygen interactions [28]. More recent applications have focused on the refinement of $\mathrm{Ca}^{2+}$ force field parameters [29, 30], energetics of $\mathrm{Ca}^{2+}$ binding in EF-hand containing proteins [31, 32, 33], and effects of $\mathrm{Ca}^{2+}$ binding on protein function $[34,35,36]$.

Elkins et al. [17] presented one of the first computational studies of PV to leverage molecular simulations. In this computational mutagenesis study, the residue in the ninth position (i.e., E59) of the silver hake (SHPV-B) PV proteins' first EF hand was mutated to arginine/serine/cysteine in order to demonstrate that the pairing of amino acids comprising the first and second $\mathrm{EF}$ hands modulated the proteins' apparent $\mathrm{Ca}^{2+}$ affinity. Cates et al. extended these studies to probe the relationship between coordinating ligands of the EF hand loops and the overall flexibility and plasticity of pike PV via MD simulations [37] to show that the EF hands inherent rigidity is preventing bidentate ligation when the residue is mutated to aspartate. More recently, Kucharski et al. utilized MD simulations and a statistical mechanical model, mean spherical approximation (MSA), to rationalize $\beta$-parvalbumin ( $\beta \mathrm{PV}) \mathrm{s}$ ' thermodynamic preference for $\mathrm{Ca}^{2+}$ over $\mathrm{Mg}^{2+}$ and concluded that EF hand rigidity disfavors binding of the smaller $\mathrm{Mg}^{2+}$ ion and thereby contributes to its selectivity for $\mathrm{Ca}^{2+}[38]$. Other studies have used methods to model the structure of EF hand motifs in PVs [39] and probe the folding/unfolding in $\beta \mathrm{PV}$. To date, no such studies have been conducted for the high affinity $\alpha \mathrm{PV}$ variants. 
In this study, we apply MD simulation and MSA approaches developed in [38] to rationalize trends in $\mathrm{Ca}^{2+}$ binding among $\alpha \mathrm{PV}$ variants and general constructs. We considered variants that exhibit strongly differing $\mathrm{Ca}^{2+}$ affinities, including two $\alpha \mathrm{PV}$ variants (S55D/E59D $\alpha \mathrm{PV}$ and D94S/G98E $\alpha \mathrm{PV}$ ) with mutations within their $\mathrm{Ca}^{2+}$ binding loops, as well as a truncated variant, $\Delta \alpha \mathrm{PV}$, which lacks two helices (A,B). Our studies indicate that while features intrinsic to the EF hands strongly contribute to $\mathrm{Ca}^{2+}$ binding and selectivity in $\alpha \mathrm{PV}$, allosteric changes accompany the observed affinity changes.

\section{Results}

Our primary goal in this study was to determine whether molecular simulations could provide mechanistic insights into the $\mathrm{Ca}^{2+}$ binding affinities and selectivities versus $\mathrm{Mg}^{2+}$ in $\alpha \mathrm{PV}$. Towards this end, we performed nearly $\approx 36 \mu$ s of unbiased MD simulations for the $\mathrm{Ca}^{2+}$-bound, $\mathrm{Mg}^{2+}$-bound and apo states of $\alpha \mathrm{PV}$, as well as several constructs (S55D/E59D $\alpha \mathrm{PV}$, D94S/G98E $\alpha \mathrm{PV}$, and $\Delta \alpha \mathrm{PV}$ ), for which structures have been reported in the literature $[15,16,18,40]$. Since atomistic resolution structural data have only been determined for a subset of these variants, we first compare our predicted structures with those available in the Protein Databank. We then test whether scores estimated via MM/GBSA and MSA theory can rank the $\alpha \mathrm{PV}$ structures in a manner consistent with their experimentally-determined $\mathrm{Ca}^{2+}$ affinities. We next decompose these scores into contributions from the EF-hand $\mathrm{Ca}^{2+}$ coordination sites versus structural and energetic changes elsewhere in the protein.

\subsection{Comparison of simulated $\alpha \mathrm{PV}$ structures}

We first verify that the MD predicted structures resemble those deposited in the Protein Databank. In Fig. 1A,B we demonstrate that the predicted apo (blue) and holo (red) WT structures are generally consistent with their respective experimental reference structures (PDB:2JWW [41] and PDB:1RWY [40] (green)). The similarity is also evidenced by the small root mean squared deviations (RMSD) values of roughly 2.0 and 1.0 $\AA$ for the apo and holo states, respectively (Fig. S1). We note similar secondary structure content in the $\alpha \mathrm{PV}$ variants relative to the wild-type (WT), including the $\beta$-sheets linking the $\mathrm{CD}$ and EF domain loops as well as the high $\alpha$-helical content (see sub-panel in Fig. 1A).

We next evaluated in Fig. 1 the per residue conformational dynamics of the proteins via root mean squared fluctuations (RMSF) to identify potential differences among the $\alpha \mathrm{PV}$ variants that could correlate with ion affinity. The RMSFs indicate that bound $\mathrm{Ca}^{2+}$ in the holo states expectedly suppress fluctuations in all variants relative to the apo states. Similar to observations in other closely related Ca-binding proteins (CBPs) including troponin $\mathrm{C}(\mathrm{TnC})[36,42]$ and $\beta \mathrm{PV}$ [38], the reduction in RMSF upon ion binding is largely restricted to the EF hand loops. Here, the bound ions stabilize the CD (residues L50 to E60) and EF (residues L85 to G95) loop regions (Fig. 1). The most significant RMSF changes occur in the EF site loop, as the average maximal peak height in the EF site loop in the apo forms is $\approx 5 \AA$ compared to $\approx 1.5 \AA$ for the 

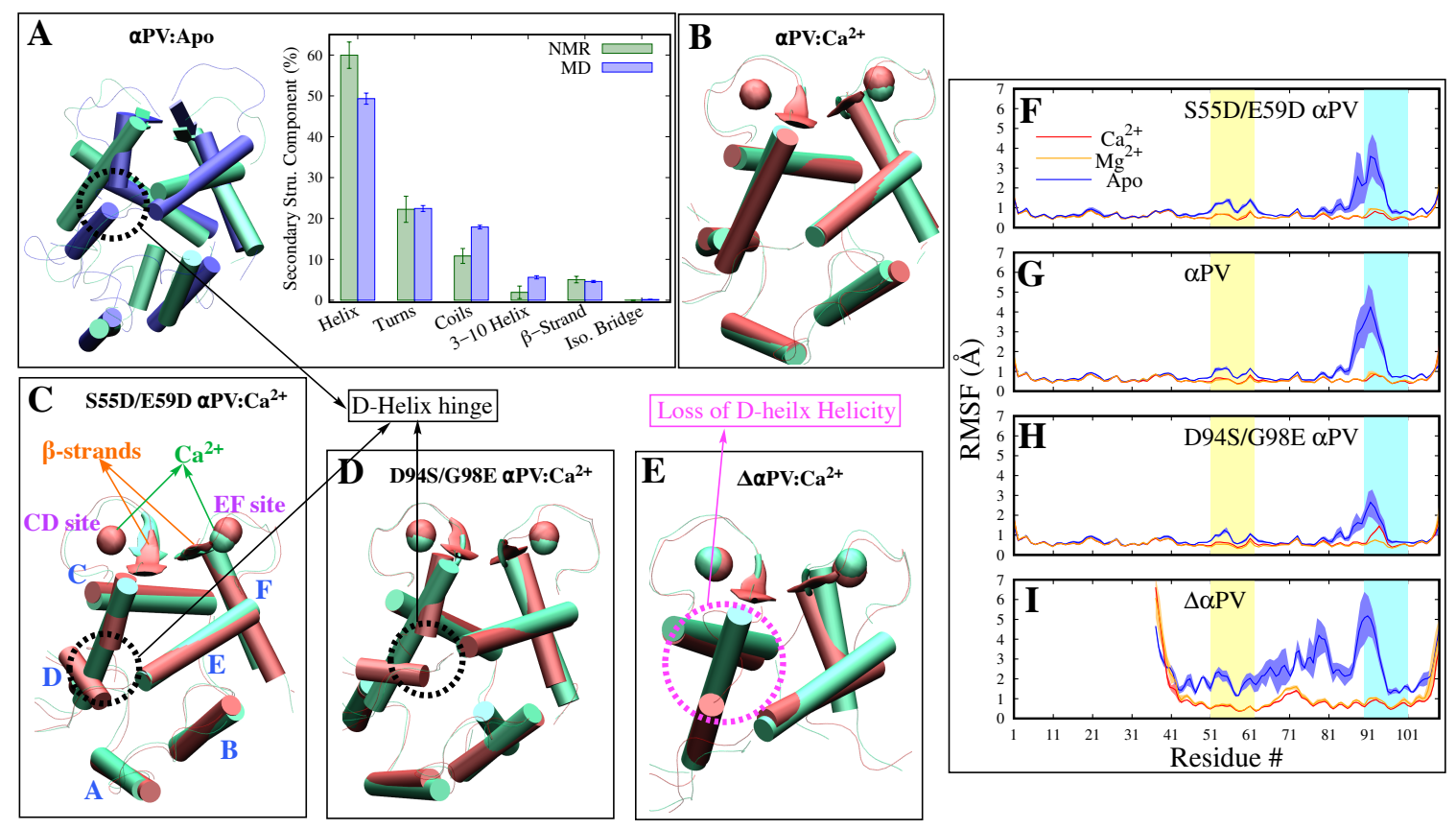

Figure 1: A-E Comparison of experimental (NMR/X-ray) and molecular dynamics (MD) predicted $\alpha \mathrm{PV}$ structures. All experimental structural snapshots are shown in green and MD snapshots are shown in either blue (apo) or red (holo). A-E Representative snapshots of NMR and MD $\alpha \mathrm{PV}$ apo systems were superposed and their secondary structural components are shown. Each MD data point is the average and standard deviation calculated from the means of three trials and the NMR data point is the average and standard deviation of all NMR modes. F-I RMSF versus residue number. All apo are colored blue and $\mathrm{Ca}^{2+}$ bound systems red and $\mathrm{Mg}^{2+}$ bound orange. Average of three trials of each system is shown; standard deviation is shown as shaded curves. Yellow and cyan shaded regions are CD and EF site loop regions. Residues spanning the different parts of the protein are mapped as follows: Helix A (S1-A17), helix B (D25-G34), helix C (A40-L50), helix D (E59-F70), CD site loop (D51-I58), helix E (S78-D90), helix F (V99-E108), and EF loop (K91-G98).

holo. Further, we noted a modest correlation between the apo-state RMSF values and $\mathrm{Ca}^{2+}$ affinity in the $\mathrm{CD}$ and EF sites, as variants with stronger $\mathrm{Ca}^{2+}$ yielded larger values relative to those with weaker affinities. We did not observe any statistical difference between the $\mathrm{Ca}^{2+}$ and $\mathrm{Mg}^{2+}$ bound forms (Fig. 1F-I). Overall, these data indicate modest correlation between the extent by which RMSFs are reduced upon $\mathrm{Ca}^{2+}$ binding and experimentally-measured binding affinities. However, as a whole, RMSF differences were small across all variants irrespective of the type of bound ion.

\subsection{Scoring $\alpha \mathrm{PV}$ variants' binding affinites}

Our simulations suggest that ion binding reduces the RMS fluctuations intrinsic to each $\alpha \mathrm{PV}$ apo state. Here we used the end-point thermodynamics method, MM/GBSA, to determine energetic bases for these changes that may contribute to high $\mathrm{Ca}^{2+}$ and $\mathrm{Mg}^{2+}$ affinity. We first tested whether energies predicted from the simulations via the MM/GBSA method could reliably rank-order the variants by their experimentally- 
determined affinities. In Table S1 we provide full MM/GBSA energies representing the internal, van der waals, electrostatic, and polar- and non-polar solvation interaction terms as free energies $\left(\mathrm{G}_{\text {Total }}^{\text {Calc }}\right)$ or $\Delta \mathrm{G}_{\text {Total }}^{\text {Calc }}$ between the $\alpha \mathrm{PV} / \mathrm{Ca}^{2+}$ complex (holo) and apo structure. Note our $\Delta \mathrm{G}_{\text {Total }}^{\text {Calc }}$ does not include contributions for the solvation energy of the free ions, as these terms are the same for each $\alpha \mathrm{PV}$ variant. To compare variants, we report $\Delta \Delta G \equiv \Delta G_{i}-\Delta G_{j}$, which cancels out contributions from the free $\mathrm{Ca}^{2+} \mathrm{s}^{\prime}$ solvation energy. The estimated MM/GBSA scores $\left(\Delta \mathrm{G}_{\text {Total }}^{\text {Calc }}\right)$ unfortunately failed to correctly rank the proteins, which we attributed to the MM/GBSA estimates for the ion/protein interactions, as explained below.

The Generalized Born Surface Area approach used for the MM/GBSA scoring was developed as a fast alternative to computing electrostatic energies from Poisson-Boltzmann (PB) theory [43]. However, standard PB theory suffers from inaccuracies when applied to highly charged systems [44], such as the $\mathrm{Ca}^{2+}$-bound EF-hand loops we considered here. Therefore, we determined if omitting the $\mathrm{Ca}^{2+} /$ protein binding interaction terms would provide a more accurate ranking, by assessing the 'reorganization score' of the protein upon binding an ion. We define this reorganization score as the difference in MM/GBSA score of the isolated protein in its holo state versus that of its apo form. Indeed, the reorganization energies yielded $\Delta \Delta \mathrm{G}_{R E}^{C a l c}$ values of $-19.94 \pm 0.82,0.0 \pm 0.84$, and $30.04 \pm 0.84 \mathrm{kcal} / \mathrm{mol}$ for S55D $/ \mathrm{E} 59 \mathrm{D} \alpha \mathrm{PV}, \alpha \mathrm{PV}$, and D94S/G98E $\alpha \mathrm{PV}$, respectively (Table 1), which suggest that forming the holo state in S55D/E59D $\alpha \mathrm{PV}$ incurs a smaller thermodynamic penalty than the WT, while $\Delta \alpha \mathrm{PV}$ incurs an even greater cost. The trend of reduced reorganization score correlates with the experimental affinity estimates of $\left(\Delta \Delta \mathrm{G}^{\text {Expt }}\right)$ of $-2.0 \pm 0.22$, $0.0 \pm 0.08$ and $3.8 \pm 0.22 \mathrm{kcal} / \mathrm{mol}$ (Table 1). We further computed a $\Delta \Delta \mathrm{G}_{R E}^{C a l c}$ of $-0.26 \pm 0.76 \mathrm{kcal} / \mathrm{mol}$ for the $\Delta \alpha \mathrm{PV}$. However, the apo $\Delta \alpha \mathrm{PV}$ structures we simulated remained partially folded and thus did not represent the unfolded state suggested by circular dichroism experiments [18], thus its $\Delta \Delta \mathrm{G}_{R E}^{\text {Calc }}$ was not meaningful. For this reason, in subsequent analyses of $\Delta \alpha \mathrm{PV}$ we only consider the score decomposition of its holo state (i.e., $\mathrm{G}_{R E}^{C a l c}$ in Table 1). We observed roughly similar trends for $\mathrm{Mg}^{2+}$, though D94S/G98E $\alpha \mathrm{PV}$ binds the ion with a modestly higher score relative to the WT. Importantly, we note that $\Delta \mathrm{G}_{R E}^{C a l c}(W T, C a)=128.78 \pm 0.59$ $\mathrm{kcal} / \mathrm{mol}$ versus $\Delta \mathrm{G}_{R E}^{\text {Calc }}(W T, M g)=248.66 \pm 0.61 \mathrm{kcal} / \mathrm{mol}$. These scores suggest that binding $\mathrm{Mg}^{2+}$ manifests in an even greater reorganization penalty relative to $\mathrm{Ca}^{2+}$ that would disfavor the binding of magnesium.

\section{$3.3 \quad \mathrm{Ca}^{2+}$ affinity determinants within the EF hands}

Since both site-directed mutations in S55D/E59D $\alpha \mathrm{PV}$ and D94S/G98E $\alpha \mathrm{PV}$ reside within the EF hands, it was expected that changes in binding free energy could be explained by differences in the binding loop conformations that coordinate $\mathrm{Ca}^{2+}$. We therefore measured the radial distribution of protein oxygens around bound ions in the $\mathrm{CD}$ site to assess the loops' coordination of $\mathrm{Mg}^{2+}$ and $\mathrm{Ca}^{2+}$ for the $\mathrm{S} 55 \mathrm{D} / \mathrm{E} 59 \mathrm{D} \alpha \mathrm{PV}(\mathrm{red})$, $\alpha \mathrm{PV}$ (green), D94S/G98E $\alpha \mathrm{PV}$ (blue) and $\Delta \alpha \mathrm{PV}$ (cyan) proteins in Fig. 2. We observed that the radial distribution maxima for the $\alpha \mathrm{PV}$ variants occurred at smaller $\mathrm{Ca}^{2+}$ /oxygen distances $(\approx 2.2 \AA)$ relative to that of the WT $(\approx 2.4 \AA$ ) (Fig. $2 \mathrm{~A})$. However, the cumulative oxygen density (Fig. 2C) obtained by integrating 
Table 1: Estimation of MM/GBSA reorganization energies.

\begin{tabular}{lrrrrr}
\hline System & $\mathrm{G}_{R E}^{\text {Calc }}($ holo $)$ & $\Delta \mathbf{G}_{R E}^{\text {Calc }}$ & $\Delta \Delta \mathbf{G}_{R E}^{\text {Calc }}$ & \multicolumn{1}{c}{$\Delta \Delta \mathbf{G}^{\text {Expt }}$} \\
\hline S55D/E59D $\alpha \mathrm{PV}: \mathrm{Ca}^{2+}$ & & $108.83 \pm 0.57$ & $-19.94 \pm 0.82$ & $-2.0 \pm 0.22$ & {$[15,45,14]$} \\
$\alpha \mathrm{PV}: \mathrm{Ca}^{2+}$ & $(-1672.66 \pm 0.42)$ & $128.78 \pm 0.59$ & $0.0 \pm 0.84$ & $0.0 \pm 0.08$ & {$[15,45,14]$} \\
& $(-1239.54 \pm 0.51 *)$ & & & & \\
& & $158.82 \pm 0.59$ & $30.04 \pm 0.84$ & $3.8 \pm 0.22$ & {$[16]$} \\
D94S/G98E $\alpha \mathrm{PV}: \mathrm{Ca}^{2+}$ & $(-1257.09 \pm 0.34)$ & $128.52 \pm 0.48$ & $-0.26 \pm 0.76$ & 9.5 & {$[18]$} \\
$\Delta \alpha \mathrm{PV}: \mathrm{Ca}^{2+}$ & & $(415.57 \pm 0.55)$ & & & \\
& & $\left(-18 \pm 0.59^{*}\right)$ & & & \\
& & $230.34 \pm 0.66$ & $-18.32 \pm 0.90$ & $-0.7 \pm 0.23$ & {$[15,45,14]$} \\
S55D/E59D $\alpha \mathrm{PV}: \mathrm{Mg}^{2+}$ & $(-1552.78 \pm 0.46)$ & $248.66 \pm 0.61$ & $0.0 \pm 0.80$ & $0.0 \pm 0.14$ & {$[15,45,14]$} \\
$\alpha \mathrm{PV}: \mathrm{Mg}^{2+}$ & & $247.01 \pm 0.60$ & $-1.65 \pm 0.86$ & $2.8 \pm 0.31$ & {$[16]$} \\
D94S/G98E $\alpha \mathrm{PV}: \mathrm{Mg}^{2+}$ & $(-1134.73 \pm 0.41)$ & $250.88 \pm 0.53$ & $2.22 \pm 0.82$ & $\mathrm{nb}$ & {$[18]$} \\
$\Delta \alpha \mathrm{PV}: \mathrm{Mg}^{2+}$ & & $(418.05 \pm 0.61)$ & & & \\
\end{tabular}

Estimates for $\Delta G_{R E}^{C a l c}=G_{R E}^{C a l c}($ holo $)-G_{R E}^{C a l c}($ apo $) . G_{R E}^{C a l c}($ holo $)$ (or) $G_{R E}^{C a l c}($ apo) are estimated as the sum of all energies of the receptor. $\Delta \Delta G_{R E}^{C a l c}$ and $\Delta \Delta G^{E x p t}$ are calculated and experimental free energies, relative to WT. Estimates considering just the holo states are shown as parenthesis. Calculated values are averages estimated from three MD trials and error bars represent standard error of mean (sem). See Table S1 for MM/GBSA energies estimated that include solvation energies. ${ }^{*}$ Energies from $\mathrm{C}-\mathrm{F}$ domains, i.e., C-F helices. ${ }^{a}$ non-binder.

the radial distributions with respect to distance (Fig. 2A) indicate that within $3 \AA$, the WT has a near optimal coordination number of eight oxygens, while the D94S/G98E $\alpha \mathrm{PV}$ and $\Delta \alpha \mathrm{PV}$ cumulative densities reflect seven bound oxygens. Curiously, the high affinity S55D/E59D $\alpha \mathrm{PV}$ variant bound $\mathrm{Ca}^{2+}$ with just six oxygens (Fig. 2C). We observed similar trends in the EF site, namely that the corresponding cumulative distributions indicate D94S/G98E $\alpha \mathrm{PV}$ coordinates seven oxygens within $3.0 \AA$ of $\mathrm{Ca}^{2+}$, whereas for all other systems only six are coordinated (Fig. 2D). Assessments for $\mathrm{Mg}^{2+}$ are summarized in Section S8.4. These lack of consensus in these data suggest that the protein coordination numbers alone do not strongly correlate with affinity. Similar analyses enumerating the waters complexed within the EF hands were also inconclusive.

The $\Delta \Delta G$ values from MM/GBSA correctly rank the $\alpha \mathrm{PV}$ proteins by their reorganization energies but only did so by neglecting $\mathrm{Ca}^{2+} /$ protein interactions as explained earlier. We therefore used an alternative method, Mean spherical approximation (MSA) theory, to estimate the thermodynamics of binding $\mathrm{Ca}^{2+}$ and other ionic species to the EF hands in a manner that accounts for the highly-charged and finite-sized atoms in the binding site. Specifically, we use MSA to relate the MD-predicted ion coordination data (numbers of coordinating oxygens and binding site volumes) to the chemical potentials for partitioning solvent ions (potassium $\left(\mathrm{K}^{+}\right), \mathrm{Mg}^{2+}, \mathrm{Ca}^{2+}$ and chloride $\left(\mathrm{Cl}^{-}\right)$) into the EF and $\mathrm{CD}$ binding domains. We present in Fig. 3 MSA-predicted chemical potentials for cations in a $150 \mathrm{mM} \mathrm{KCl,} 1 \mathrm{mM} \mathrm{MgCl} \mathrm{M}_{2}$ and $2 \mu \mathrm{M} \mathrm{CaCl}_{2}$ solution that emulate intracellular ionic strengths. The chemical potentials are presented for several binding site volumes to reflect the range of maxima in the oxygen radial distributions we computed $\left(0.4\right.$ to $0.65 \mathrm{~nm}^{3}$ to represent radii of 0.25 to $0.36 \mathrm{~nm}$ ) as well as coordination by five to eight oxygens. Additionally, we varied the average charge of the coordinating oxygens to reflect the contributions of backbone oxygens (-0.57e), serine hydroxyl oxygens (-0.65e), glutamic or aspartic acid oxygens (-0.82), and water oxygens (-0.83e) to the average effective charge of oxygen in the binding domain. Our MD simulation data indicate an effective 
oxygen charge $\left(q_{\mathrm{eff}}\right)$ of the CD site for the S55D/E59D $\alpha \mathrm{PV}$ variant of $-0.77 \mathrm{e}$, which is more negative than the CD site for the other variants. This difference reflects a greater proportion of oxygens from acidic side chains in the CD site of S55D/E59D $\alpha \mathrm{PV}$ (while the EF-hands for all cases have $q_{\mathrm{eff}}=-0.75 \mathrm{e}$ (see Table S6)).

Our results in Fig. 3 consistently demonstrate that the chemical potentials for $\mathrm{Ca}^{2+}$ (red) are more favorable than $\mathrm{Mg}^{2+}$ (blue) by approximately $2 \mathrm{kcal} / \mathrm{mol}$ over all binding site radii we considered. This difference in large part reflects the higher desolvation energy of $\mathrm{Mg}^{2+}$ relative to $\mathrm{Ca}^{2+}$ (-1931.4 versus 1608.3 $\mathrm{kJ} / \mathrm{mol}[46]$ ) that offsets the entropic advantage of partitioning the smaller ion into the binding site. This result is consistent with experimental data indicating that $\mathrm{Ca}^{2+}$ affinities are $\approx 10 \mathrm{kcal} / \mathrm{mol}$ (see Tables $\mathrm{S} 1$, 1) more favorable than $\mathrm{Mg}^{2+}$. Further, binding site configurations with a more prominent negative charge (-0.77e), e.g. for S55D/E59D $\alpha \mathrm{PV}$ CD site, presented modestly more favorable chemical potentials for divalent cations of roughly $0.5 \mathrm{kcal} / \mathrm{mol}$ over all other variants (-0.75e). In other words, the more negative CD binding site of $\mathrm{S} 55 \mathrm{D} / \mathrm{E} 59 \mathrm{D} \alpha \mathrm{PV}$ provides thermodynamic stability over the other site configurations and is consistent with the $\approx 2 \mathrm{kcal} / \mathrm{mol}$ (see Table S1) energy difference reported via experiment. Further, the model indicates that the chemical potential is modestly insensitive to the number of bound oxygens ( 8 is favored over 5 by less than $1 \mathrm{kcal} / \mathrm{mol}$ ). Since MSA is a coarse approximation of ion binding thermodynamics, it is unclear whether $<1 \mathrm{kcal} / \mathrm{mol}$ difference in chemical potentials is significantly different for $\alpha \mathrm{PV}$. Nonetheless, by accounting for finite size effects and competition with other solvated electrolytes for the proteins' binding domains via MSA theory, the proteins' $\mathrm{Ca}^{2+}$ selectivity over $\mathrm{Mg}^{2+}$ and modest differences in the predicted $\mathrm{Ca}^{2+}$ chemical potentials can be rationalized. In contrast, the MM/GBSA scores summarized in Section S8.4 suggest that the MM/GBSA $\Delta \Delta \mathrm{G}_{R E}^{C a l c}$ reorganization scores for the CD site, but not the EF site, correlated with the variants' $\mathrm{Ca}^{2+}$ affinities (Table $\mathrm{S} 2$ ).
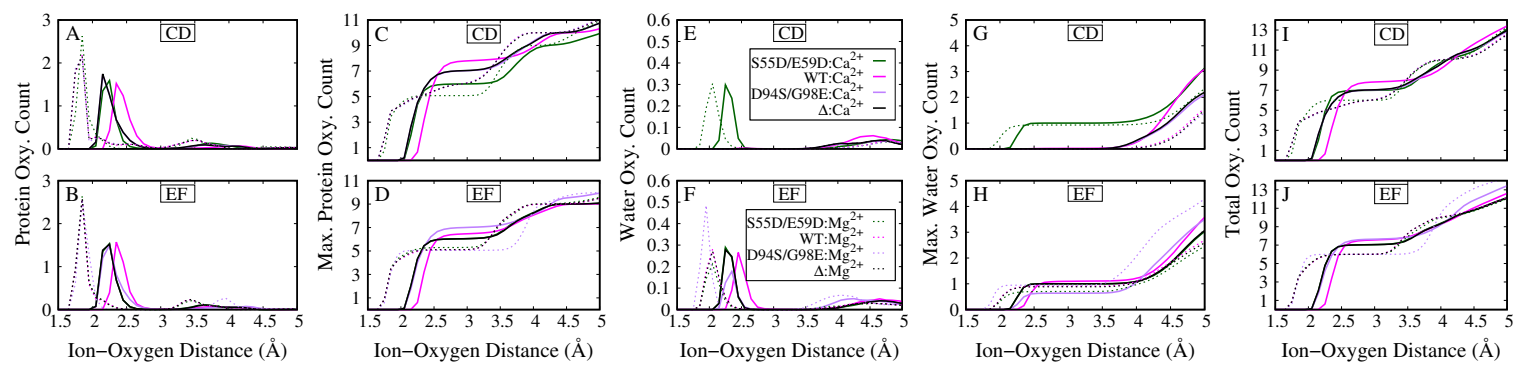

Figure 2: Protein and water co-ordination with the ions in the CD and EF sites. A, B Radial distribution of protein oxygens in the CD (E) and EF (F) sites. C, D Maximal amino acid oxygen count in $\mathrm{CD}(\mathrm{G})$ and EF $(\mathrm{H})$ sites. E, F Radial distribution of water oxygens in the $\mathrm{CD}(\mathrm{A})$ and EF (B) sites. G, $\mathbf{H}$ Maximal water oxygen count in CD (C) and EF (D) sites. I, J Total oxygen count, i.e., the sum of water and amino acid oxygen count in $\mathrm{CD}(\mathrm{C})$ and EF (D) sites. All $\mathrm{Ca}^{2+}$ bound systems are shown as full lines and the $\mathrm{Mg}^{2+}$ bound ones as broken lines. S55D/E59D $\alpha \mathrm{PV}, \alpha \mathrm{PV}$, D94S/G98E $\alpha \mathrm{PV}$ and $\Delta \alpha \mathrm{PV}$ are colored in green, magenta, purple and black respectively. 


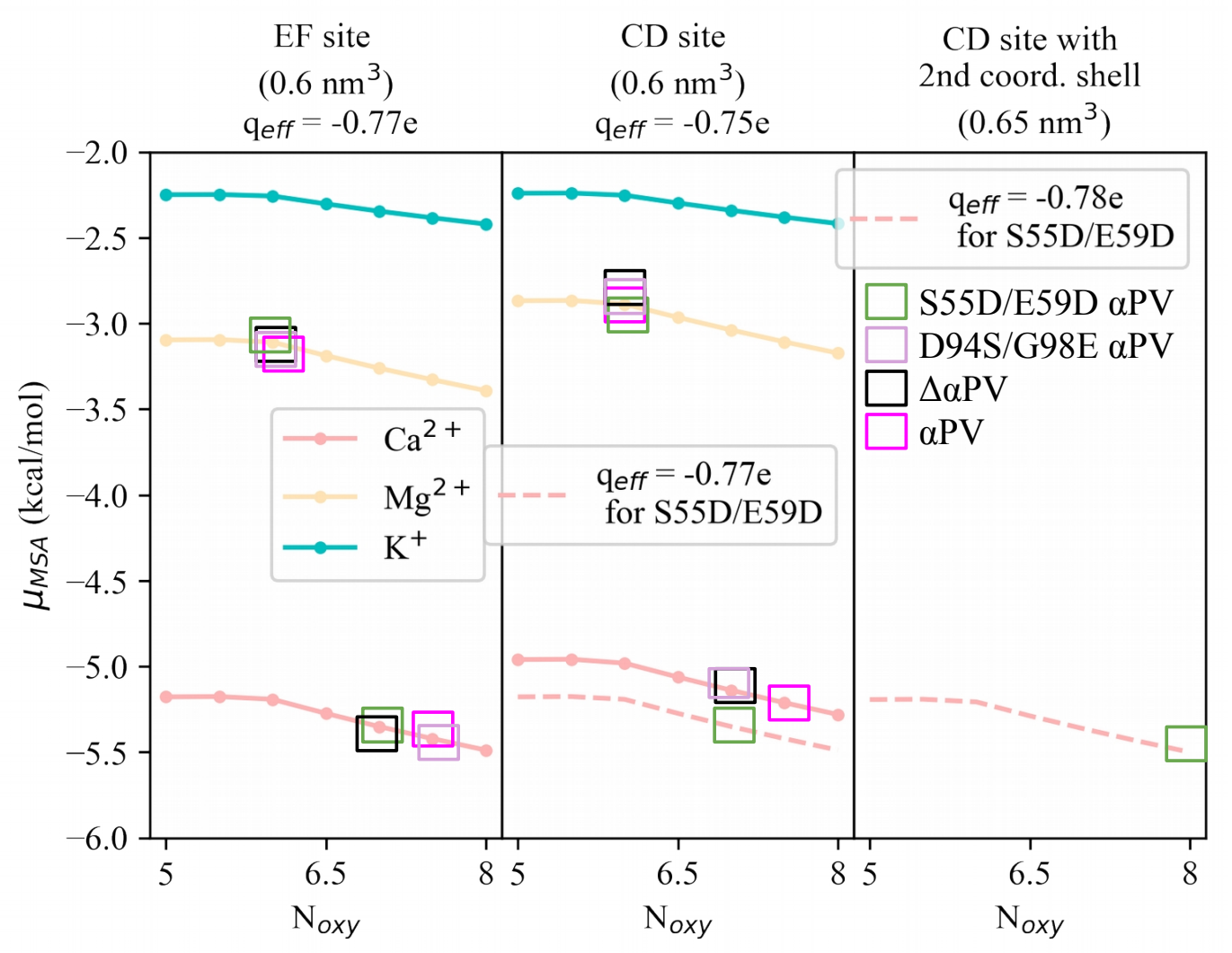

Figure 3: MSA free energies for cations in the $\alpha \mathrm{PV}$ binding site with bath concentrations $[\mathrm{KCl}]=150 \mathrm{mM}$, $\left[\mathrm{MgCl}_{2}\right]=1 \mathrm{mM}$ and $\left[\mathrm{CaCl}_{2}\right]=1 \mu \mathrm{M}$ under varying numbers of coordinating oxygens $\left(N_{\text {oxy }}\right)$ from 5.0 to 8.5. The remaining parameters are the same as our previous MSA calculations in [38]. The colored boxes indicate the $\alpha \mathrm{PV}$ systems we simulated (including water oxygens).

\subsection{Ion affinity determinants beyond the EF hands}

We have so far demonstrated that MD-predicted differences in the EF-hand coordination and associated reorganization energies among the $\alpha \mathrm{PV}$ variants loosely correlate with experimental measurements. Nevertheless, we found that point mutations also impart changes in the overall structure of the protein (Fig. 1B-D), as well as its dynamics (Fig. 1F-H). We therefore investigated properties of the entire protein that could further contribute to the range of affinities reported in $\alpha \mathrm{PV}$ variants, including desolvation, helix bundle 'packing', and secondary structure.

Graberek et al. [21] speculated that waters coordinated to solvated $\mathrm{Ca}^{2+}$ are released into the bulk medium as the ion binds proteins. These liberated waters increase the configurational entropy of the bulk medium and thereby lower the free energy of the system. In this vein, we investigated analogous changes in the solvation of the $\alpha \mathrm{PV}$ variants that occur during $\mathrm{Ca}^{2+}$ binding. In Fig. $4 \mathrm{C}$, we report the number of waters within $4.0 \AA$ of each protein configuration as an indicator of solvation. Across all systems, we observe at least a reduction (green bars) of twenty bound waters in the ion-bound holo states relative to the apo 
states. Moreover, there is a significantly greater loss of solvent for the intact proteins that strongly bind Ca ${ }^{2+}$ $(\sim 54, \sim 56$ for S55D/E59D $\alpha \mathrm{PV}$ and $\alpha \mathrm{PV})$ relative to D94S/G98E $\alpha \mathrm{PV}(\sim 26)$. Similar trends were observed in $\mathrm{Mg}^{2+}$ bound systems, although, no significant differences were found relative to the $\mathrm{Ca}^{2+}$ bound systems. These trends in water loss are similar to those observed for the proteins' radii of gyration (Fig. $4 \mathrm{~A}) . \Delta \alpha \mathrm{PV}$ is an exception to this trend in that at least 50 water molecules were liberated; in all likelihood, this number would be far greater if the unfolded ion-free state were considered. The changes in water solvation appear to arise from a reduction in the proteins' solvent accessible surface area (SASA) upon ion binding (Fig. S3). Overall, we observe a consistent trend of increased dehydration upon ion binding that correlates with the intact proteins' increasing $\mathrm{Ca}^{2+}$ affinity.

In the previous section, we identified correlated differences in desolvation and SASA upon ion binding. We speculated that these differences could be attributed to isoform-specific configurations of the helices composing the proteins globular domains. We therefore report in Fig. 1B-E the relative positions of helices composing the proteins' hydrophobic core in order to highlight conformational changes among the variants as they bind $\mathrm{Ca}^{2+}$. These changes culminated in more compact proteins during ion binding as evidenced by the reduction of their radii of gyration (Fig. 4). We anticipated these structural changes reorganize the interfaces between bundle helices, and entail reorganization of the hydrogen bonding network within and between these helices. Along these lines, we demonstrate that the number of hydrogen bonds tend to be greater in the holo state relative to apo (green bars in Fig. 4D). However, only for S55D/E59D $\alpha \mathrm{PV}$ was the change significantly different from zero among the intact proteins. Hence it was apparent that more side chain hydrogen bonds were formed than broken during ion binding, but we did not observe consistent patterns that strongly differentiated weak from strong $\mathrm{Ca}^{2+}$ binders. Further, although the average change in hydrogen bond appears to be lower for the $\mathrm{Mg}^{2+}$ bound systems, the difference between the two ions is not significant. A summary of specific hydrogen bonding interactions involving side chains is provided in Section S8.4. Overall, we show that upon ion binding increased hydrogen bonding resulting in an overall decrease in the reorganization scores as shown in Table 1.

In Fig. $4 \mathrm{D}$ we note that the total number of hydrogen bonds in $\Delta \alpha \mathrm{PV}$ is significantly smaller $(\approx 35)$ compared to the intact systems $(\approx 60)$ as would be expected from loss of its AB helices. Interestingly, the change in hydrogen bonds we report for $\Delta \alpha \mathrm{PV}$ is significantly greater than zero $(\approx 6)$, which suggests $\mathrm{Ca}^{2+}$ plays an important role in increasing the connectivity of the hydrogen bond network within the CDEF helices. We expect that the actual change in the number of hydrogen bonds would be far greater if we had assumed an unfolded conformation for the apo state. From this set we identified seven hydrogen bonds spanning the $\mathrm{AB} / \mathrm{CDEF}$ interface that underwent significant changes in bonding frequency during ion binding (see Fig. 4B). Bonds that exhibited greater contact frequency upon $\mathrm{Ca}^{2+}$ binding include F18/R75, D22/E81, D22/K80, D22/S78, S23/E81, and F24/E81, while K36/S109 had fewer contacts. Though nearly all of these reflected increases in bonding frequency in the holo relative to apo states, those changes were generally minor. In other words, the hydrogen bonding network was modestly 'tightened' relative to its apo configuration, as 
opposed to undergoing dramatic changes in the hydrogen bond network. These structural changes culminated in a net gain of $\approx 18 \mathrm{kcal} / \mathrm{mol}$ upon deleting the $\mathrm{AB}$ domain from $\alpha \mathrm{PV}$ (Table 1 and Fig. S4), as assessed using scores for residues K37/M37 to E108 in $\alpha \mathrm{PV}$. Hence, it is apparent that there are hydrogen bonds between $\mathrm{AB} / \mathrm{CDEF}$ helices in the WT $\alpha \mathrm{PV}$ that are favorable and undoubtedly help to stabilize the folded state. Some of these appear to have higher bonding frequencies, which may imply the AB helices help to stabilize the hydrogen bonds and other nearby residues in the holo over apo states.

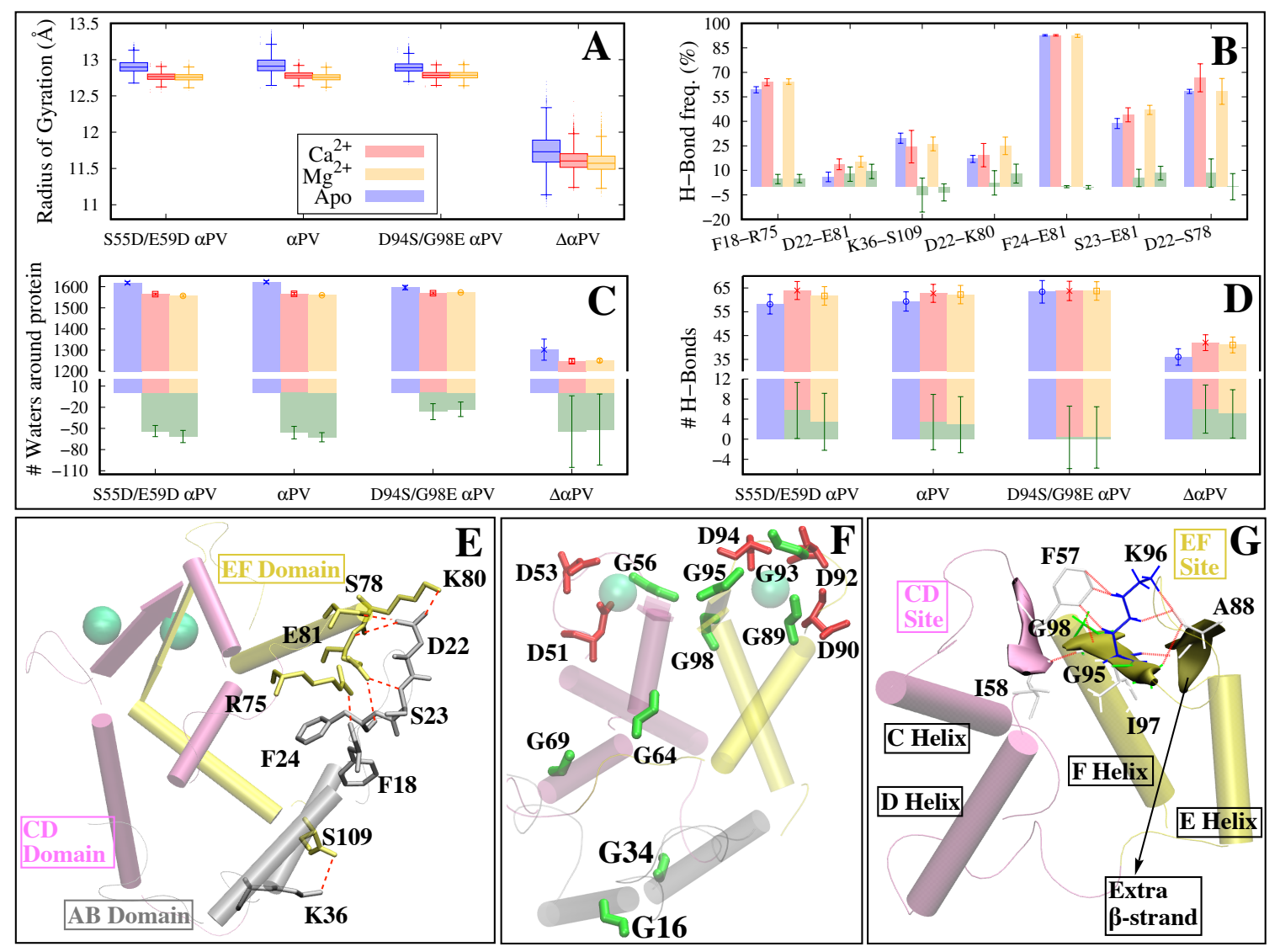

Figure 4: A Comparison of radii of gyration $\left(\mathrm{R}_{g}\right)$ of all systems. All three trails of a system are plotted as a single box plot. Boxes represent the 25 th to 75 th quartile (i.e., the interquartile range where $50 \%$ of data is concentrated), bottom and top whiskers are minima and maxima, and the horizontal line in the boxes represent the median, and the points above and below whiskers are outliers. B, E Hydrogen bonds that are formed between the $\mathrm{AB}$ domain (colored silver) and remainder of the protein in the holo $\alpha \mathrm{PV}$. CD and EF domains are colored mauve and yellow, respectively. C Waters within $4 \AA$ of protein. Each data point represents the mean of three trials and error bar represent standard deviation. Green bars are the difference between holo and apo systems $(\Delta \mathrm{W})$. D Total number of hydrogen bonds in the last 200ns of simulation. Error bars are standard deviations estimated from three MD trials. Green bars are the difference between holo and apo systems $(\Delta \mathrm{HB})$. F Glycines (green) and aspartic acids (red) in $\alpha \mathrm{PV}$. G Extra $\beta$-strand formed in the EF loop (colored yellow) in the apo $\Delta \alpha \mathrm{PV}$. Extra $\beta-\beta$ hydrogen bonds that are formed in the EF loop region are colored red and the corresponding residues are shown as sticks. CD and EF domains are colored pink and yellow respectively.

Given the general increase in hydrogen bonding upon complexing metals we reported in the last section, we 
next assessed changes in $\alpha$-helix and $\beta$-sheet secondary structure and their corresponding energies. $\alpha$-helices represent the most abundant secondary structure in $\alpha \mathrm{PV}(\approx 45-55 \%)$ (Fig. $1 \mathrm{~A}$ and Fig. 5A). Our MD simulations indicate that binding ions increases the $\alpha$-helical content of all $\alpha \mathrm{PV}$ variants $(\approx 3-6 \%)$, with $\mathrm{Ca}^{2+}$ to a greater extent than $\mathrm{Mg}^{2+}(\approx 1-4 \%)$ (Fig. $\left.5 \mathrm{~A}\right)$. Overall, the increase in $\alpha$-helicity is greater for $\mathrm{Ca}^{2+}$ relative to $\mathrm{Mg}^{2+}$, while the magnitude of helicity changes is inversely correlated with affinity among the intact variants (5\% for D94S/G98E $\alpha \mathrm{PV}, 4 \%$ for WT, 3\% for S55D/E59D $\alpha \mathrm{PV}$ ).

In Fig. S6 we show that most of the changes in helicity during ion binding arise in helix E (residues A79 to G89), and to a lesser extent in helix D (residues E60 to F70). These data indicate $\alpha$-helical character increases in the holo relative to the apo state and those changes are adjacent to the loop residues that directly coordinate $\mathrm{Ca}^{2+}$. For instance, the N-terminal residues of helix D (E60-S65) undergo an increase from under $50 \%$ helicity to approximately 60 and $90 \%$ for $\mathrm{Mg}^{2+}$ and $\mathrm{Ca}^{2+}$, respectively (Fig. 5C, D) and (Fig. S6) versus more modest changes within S65-F70. The low helicity of the apo state residues E60-G64 (by $\approx 20-25 \%$ in the D-helix coincides with the glycine at position 64 (shown in Fig. 4F). Similarly, the N-terminal region of helix E (A79-T84) exhibits $\approx 90 \%$ or greater $\alpha$-helicity, compared to the C-terminal end that approaches less than $60 \%$ at residue A87. These data also suggest that the binding of $\mathrm{Ca}^{2+}$ and $\mathrm{Mg}^{2+}$ maximize the helical content to nearly $100 \% \cdot \mathrm{Mg}^{2+}$ reduces the helicity of residues E60-S65 in D-helix at least by $20 \%$ in all variants (Fig. 5D). Helices D and E exhibited increased helicity upon ion binding, which in turn yielded favorable $\Delta \mathrm{G}_{R E}^{\text {calc }}$ scores that correlated with experimental trends in $\mathrm{Ca}^{2+}$ affinity (see Fig. S4 and Table S4).

We also demonstrate that truncating the $\mathrm{AB}$ domain from $\alpha \mathrm{PV}$ reduces the overall helical content, particularly within the D helix. Specifically, as with the intact variants, the greatest impact on the helicity of the D-helix was restricted to the N-terminal end (E60-S65 ), 55\% versus nearly 90\% for the intact variant (Fig. 5C). The corresponding energies from MM/GBSA indicated that the D helix was more thermodynamically favored in the WT holo $\alpha \mathrm{PV}$ relative to $\Delta \alpha \mathrm{PV}\left(\mathrm{Ca}^{2+}\right.$ bound $\alpha \mathrm{PV}$ and $\Delta \alpha \mathrm{PV}$ are $\approx-149$ and $\approx-137$ $\mathrm{kcal} / \mathrm{mol}$ respectively for helix D from Table S4). For the E helix, no differences were not apparent (Table S5). These data indicate that the $\mathrm{AB}$ domains maintain a high degree of $\alpha$-helicity in the $\mathrm{D}$ helix upon ion binding, which likely contributes to high affinity $\mathrm{Ca}^{2+}$ binding.

The two $\beta$-strands in $\alpha \mathrm{PV}$ couple the two ion binding domains through the formation of anti-parallel $\beta$-sheet (Fig. 1A). This secondary structure element is common across EF-hand containing proteins and are implicated in shaping $\mathrm{Ca}^{2+}$ binding affinity $[47,20]$. We observed in $\alpha \mathrm{PV}$ proteins that the binding of $\mathrm{Ca}^{2+}$ or $\mathrm{Mg}^{2+}$ both reduce the $\beta$-strand content in the $\alpha \mathrm{PV}$ systems by $\approx 1-5 \%$, with generally more significant reductions in $\beta$ character for $\mathrm{Ca}^{2+}$ binding relative to $\mathrm{Mg}^{2+}$ (Fig. 5B). This reduction in $\beta$-strand content likely occurs as a result of F57 (Table S7) and K96 (Table S8) backbone oxygens shifting from the $\beta$-sheet in the apo state to binding $\mathrm{Ca}^{2+}$ in the holo state. $\beta$-strand content is reduced in residues $\mathrm{F} 57-\mathrm{E} 59$ and K96-G98 most significantly by $\approx 50-60 \%$ for $\mathrm{Ca}^{2+}$ versus $\approx 20-40 \%$ for $\mathrm{Mg}^{2+}$ (Fig. S7). Additionally, we note that the reductions in $\beta$ sheet character are inversely correlated with the $\mathrm{Ca}^{2+}$ binding affinity of the $\alpha \mathrm{PV}$ variants, although the effect size by percentage is relatively small $(\approx-1.0 \%$ for $\mathrm{S} 55 \mathrm{D} / \mathrm{E} 59 \mathrm{D} \alpha \mathrm{PV}$ relative to 

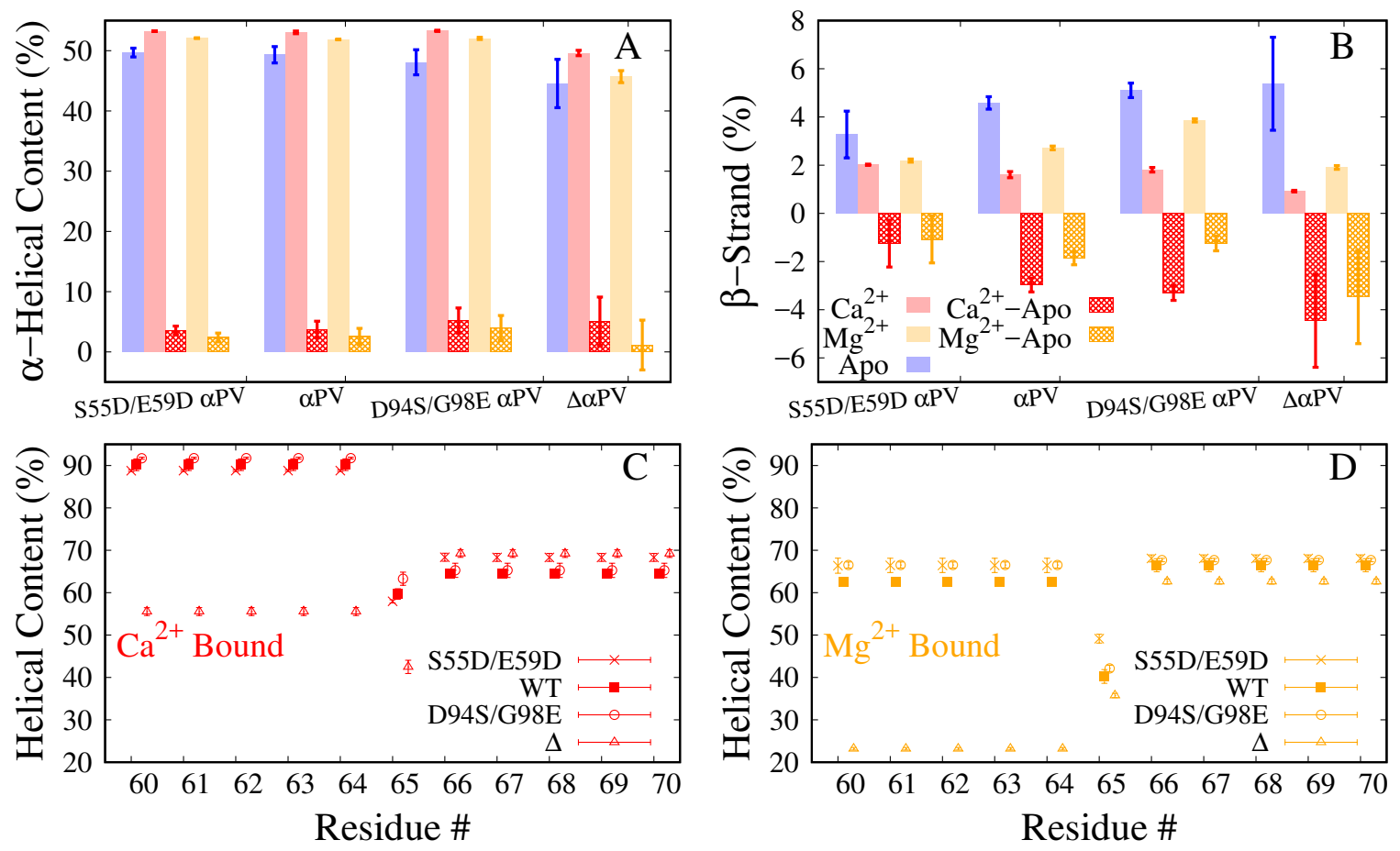

Figure 5: $\quad$ Secondary structural elements of all systems compared. A-B $\alpha$-helicity (A) and $\beta$-strand (extended configuration according to VMD) (B) of the entire protein over the entire simulation time was calculated. Each data point is the average and standard deviation of three trials of each system (taken from means of each trial). The difference between the holo state and apo state is also plotted as grid bars. Only $\mathrm{C}_{\alpha}$ atoms are considered for this calculation. $\mathbf{C}$-D $\alpha$-helix of the D-helix residues for the $\mathrm{Ca}^{2+}(\mathrm{C})$ and $\mathrm{Mg}^{2+}$ (D) bound systems are compared.

$\approx-3 \%$ for D94S/G98E $\alpha \mathrm{PV})$. We had anticipated that the more extensive unfolding of the $\beta$-strands in weaker versus stronger variants would yield thermodynamically unfavored score changes. As expected, we found that the associated changes in $\Delta \Delta \mathrm{G}_{R E}^{\text {Calc }}(\beta)$ for these residues correlated with affinity $(-17 \mathrm{kcal} / \mathrm{mol}$ for S55D/E59D $\alpha \mathrm{PV}$ versus $28 \mathrm{kcal} / \mathrm{mol}$ for D94S/G98E $\alpha \mathrm{PV}$ in Table S3) Hence, we observe potentially meaningful trends in the extents of $\beta$-sheet unfolding during ion binding that correlate with the variants' respective affinities.

\section{Discussion}

$\alpha \mathrm{PV}$ is among the highest affinity binders of $\mathrm{Ca}^{2+}$ in the $\mathrm{PV}$ family. The two site-directed mutants evaluated in our study include S55D/E59D $\alpha \mathrm{PV}$ and D94S/G98E $\alpha \mathrm{PV}$, which bind $\mathrm{Ca}^{2+}$ with stronger and weaker affinities compared to the $\mathrm{WT} \alpha \mathrm{PV}$, respectively. Both variants reflect substitutions in the $\mathrm{Ca}^{2+}$ binding EF-hand loops, which would generally be expected to directly impact $\mathrm{Ca}^{2+}$ affinity by changing the ions' coordination. A truncated $\alpha \mathrm{PV}$ variant $(\Delta \alpha \mathrm{PV})$ was also considered to investigate the role of the $\mathrm{AB}$ helices indirectly influencing the binding of $\mathrm{Ca}^{2+}$. Our primary goals of this study were to 1) test the hypothesis that 
$\mathrm{Ca}^{2+}$ affinity is significantly modulated by structural features beyond the ion-coordinating EF hand loops, 2) investigate bases governing $\mathrm{Ca}^{2+}$ binding affinities among three $\alpha \mathrm{PV}$ variants and (3) understand the factors governing the selectivity of these variants for $\mathrm{Ca}^{2+}$ over $\mathrm{Mg}^{2+}$. To accomplish these goals, MD simulations and thermodynamic analyses of ion binding were conducted of these $\alpha \mathrm{PV}$ variants. We discuss factors contributing to ion binding affinity and selectivity in order of importance. Limitations of the Methodology are provided as Supplemental Data (Section S8.5).

\subsection{Rank-ordering $\alpha \mathrm{PV}$ variants according to binding affinity}

One of our goals was to elucidate the thermodynamic factors governing the variations in affinity among variants of $\alpha \mathrm{PV}$. Free energy techniques including thermodynamic integration, umbrella sampling and adaptive biasing force have been used for rigorous estimates of $\mathrm{Ca}^{2+}$ affinity for a variety of $\mathrm{Ca}^{2+}$-binding proteins [48, 26]. These methods achieve high accuracy, i.e., the estimated free energies are within $1 \mathrm{kcal} / \mathrm{mol}$, but do so with a prohibitive computational expense [49]. Further, it is frequently difficult to decompose free energies predicted by these approaches into per-residue contributions, which limits our ability to implicate specific structural differences between variants to conformations and dynamics to their respective ion affinities. For these reasons, we determined if whether the less accurate, but more computationally-feasible MM/GBSA end-point method [49] could rank-order variants by their relative affinity differences. We found that the default strategy of including $\mathrm{Ca}^{2+} /$ protein interactions yielded energies inconsistent with the $\alpha \mathrm{PV}$ proteins' experimentally measured $\mathrm{Ca}^{2+}$ affinities, which we attribute to shortcomings in the evaluation of polar solvation energies (see Limitations). However, our results suggest focusing on the reorganization score of forming the holo state upon ion binding relative to the apo rank-orders the variants correctly.

\subsection{Contributions of EF-hand loop and co-ordination shell to altered $\mathrm{Ca}^{2+}$ bind- ing}

The CD and EF domains are the primary binding sites for $\mathrm{Ca}^{2+}$ in $\alpha \mathrm{PV}$ and form the helix-disordered loop-helix EF-hand motif. The disordered loop reorganizes to coordinate its amino acid oxygens with $\mathrm{Ca}^{2+}$, which manifests in the reduced RMS fluctuations of all $\alpha \mathrm{PV}$ variants upon ion binding predicted by our MD simulations. The most significant reductions were apparent within the EF site loop (Fig. 1F-I), which we hypothesize stems from its greater number of glycines (4) compared to the CD site loop (1). This is rationalized by noting that glycine is regarded as the most labile residue of the twenty natural amino acids [50]. Interestingly, we observed that the amplitude of the apo state RMSFs in the $\mathrm{Ca}^{2+}$ binding sites correlated with the variants' ion affinities, with the largest RMSFs reported for S55D/E59D $\alpha \mathrm{PV}$ and smallest for D94S/G98E $\alpha \mathrm{PV}$. The correlation of increased fluctuations in the apo state and $\mathrm{Ca}^{2+}$ affinity is consistent with observations of larger Stokes radii and thermal instability for variants of paramecium calmodulin, which were shown to impart increased $\mathrm{Ca}^{2+}$ binding affinity relative to the WT [51]. Similarly, Vologzhannikova et al. 
confirmed the importance of disordered apo states in conferring elevated $\mathrm{Ca}^{2+}$ affinity, as they demonstrated $\beta \mathrm{PV}-1$ apo state from coho salmon is unfolded and associated with its increased affinity compared to other $\beta \mathrm{PV}$ apo forms that are folded [52]. Especially for the CD-loop sites, the calculated $\mathrm{MM} / \mathrm{GBSA} \Delta \Delta \mathrm{G}_{R E}^{C a l c}$ scores clearly indicated that the conformational changes upon $\mathrm{Ca}^{2+}$ binding yielded more favorable values for $\mathrm{S} 55 \mathrm{D} / \mathrm{E} 59 \mathrm{D} \alpha \mathrm{PV}$ and less favorable for D94S/G98E $\alpha \mathrm{PV}$ relative to $\mathrm{WT}($ Table S2). Overall, we show that a decreasing apo state stability in intact PVs is associated with increased $\mathrm{Ca}^{2+}$ affinity.

It is tempting to interpret differences in affinities among the variants based on properties of just the $\mathrm{Ca}^{2+}$ binding loop, as studies have argued that the position and charge of amino acid substitutions can dramatically enhance $\mathrm{Ca}^{2+}$ affinity [53]. For instance, it might be expected that increasing the number of oxygens bound to $\mathrm{Ca}^{2+}$ could improve affinity, as has been rationalized for selective binding of $\mathrm{Ca}^{2+}$ over $\mathrm{Mg}^{2+}$ based on bidentate glutamate binding relative to monodentate [21, 22]. Our data instead indicate that the number of protein oxygens predicted to coordinate to the ion is uncorrelated with their affinity. Further, the position of the mutation in the EF-hand loop may also determine the extent of its impact on binding ions. For example, the E101D mutation in PV was found to reduce coordination, which we attribute to the $\mathrm{F}$ helix's limited ability to reorganize to bind $\mathrm{Ca}^{2+}$. In contrast, the greater mobility elsewhere in the EF-hand loop facilitated the reorganization of adjacent amino acids to compensate for any loss in coordination due to a given mutation. In addition, we found that bound water can directly participate in coordination through bridging an amino acid with $\mathrm{Ca}^{2+}$, which we recently demonstrated was an important factor in modulating $\mathrm{Ca}^{2+}$ affinities of the $\mathrm{Ca}^{2+}$-binding ATPase SERCA [54]. Despite the lack of correlation between coordination number and affinity, our analyses firmly indicate that loop/ion interactions represent significant contributions to the MM/GBSA-estimated $\Delta \Delta G$ s relative to the WT, which favor S55D/E59D $\alpha \mathrm{PV}$ over $\Delta \alpha \mathrm{PV}$. For the most part, the changes in $\Delta \Delta \mathrm{G}_{R E}^{C a l c}$ within each loop was dominated by the $\Delta \Delta \mathrm{G}_{R E}^{C a l c}$ at the mutation site. While the trend in the $\Delta \Delta G_{R E}^{C a l c}$ for the sites could be easily rationalized (e.g., polar-to-charged residues like S55D were favorable), the magnitudes of which, especially relative to other mutations, were difficult to surmise without the simulations. This provides evidence that coordination shell alone is just one factor the contributes to $\mathrm{Ca}^{2+}$ affinity in $\alpha \mathrm{PV}$.

We used these two methods of energetic analysis to quantify the contributions of oxygen and water coordination with $\mathrm{Ca}^{2+}$. Using the MM/GBSA analysis approach (Fig. S4) we found that the S55D mutation is thermodynamically unfavorable $(11 \mathrm{kcal} / \mathrm{mol})$, whereas the E59D mutation is favorable $(-18 \mathrm{kcal} / \mathrm{mol})$, though these calculations neglected stabilizing $\mathrm{Ca}^{2+} /$ protein interactions. Because of this, we used the MSA approach to estimate ion binding chemical potentials that account for ion binding shell volume, finite sizes of the ions and oxygens, number of coordination oxygens, as well as the ion solvation energy. The MSA approach confirmed that $\mathrm{Ca}^{2+}$ was thermodynamically favored over $\mathrm{Mg}^{2+}$ across broad binding site volumes and coordination numbers (Fig. 3). Moreover, S55D/E59D $\alpha \mathrm{PV}$ was shown to more favorably bind Ca ${ }^{2+}$ relative to the WT $(\Delta \mu=0.14 \mathrm{kcal} / \mathrm{mol})$ and D94S $/ \mathrm{G} 98 \mathrm{E} \alpha \mathrm{PV}(\Delta \mu=0.21 \mathrm{kcal} / \mathrm{mol})$ on account of its more negative oxygen effective charge $\left(\Delta q_{e f f}=-0.02 e\right)$. In general, $\mathrm{Ca}^{2+}$ affinity had a modest dependence on 
binding site volumes and coordination numbers. In other words, we speculate that the moderate variations in binding site volumes and oxygen coordination numbers we computed for the $\alpha \mathrm{PV}$ variants did not appreciably impact the $\mathrm{Ca}^{2+}$ chemical potential. In fact, we applied this to a range of PV homologs with at least $50 \%$ sequence identity to the $\mathrm{WT} \alpha \mathrm{PV}$, which included several $\beta$-parvalbumins structures. The model predicted chemical potentials for a given average effective oxygen charge varied by up to $1 \mathrm{kcal} / \mathrm{mol}$ based on the MSA method, despite the diversity of coordination numbers and binding site volumes (see Fig. S8). Instead, the most significant difference was found for PDB 2MBX and 5XND, as the two species have significantly different effective oxygen charges in the binding domain ( $-0.74 \mathrm{e}$ versus $-0.81 \mathrm{e})$. Hence, properties of the ion coordination shell alone appear to have limited ability to rationalize the affinity differences in the $\alpha \mathrm{PV}$ variants we considered, unless significant changes in the charge density were involved. Even then, consideration of factors like changes in configurational entropy of the loop and strain induced within the loop upon ion binding are likely to significantly influence ion binding thermodynamics [38].

\subsection{Contributions of protein structure beyond EF-hand loop in determining $\mathrm{Ca}^{2+}$ binding}

Our analyses of the $\mathrm{Ca}^{2+} / \mathrm{EF}$-hand binding thermodynamics indicate that there is a strong driving force to optimize $\mathrm{Ca}^{2+}$ coordination, which is afforded through the ease with which the EF-hands can reorganize. Although this reorganization appears to normalize $\mathrm{Ca}^{2+}$ binding properties within the loops, the respective changes in loop conformations can drive compensatory adjustments to the $\alpha \mathrm{PV}$ helical bundle. This coupling between EF-hand binding and allosteric changes in bundle organization is exemplified in $\mathrm{Ca}^{2+}$ binding proteins such as calmodulin $[55,56]$, TnC $[57,58]$ and S100A1 [59, 60, 61]. The openings of these proteins' hydrophobic peptide-binding domains following $\mathrm{Ca}^{2+}$ binding are generally not evident in the $\mathrm{Mg}^{2+}$-bound complexes. We show that the enhanced binding of $\mathrm{Ca}^{2+}$ to $\alpha \mathrm{PV}$ protein correlates with several structural changes beyond the EF-hand loop region. The predominant changes caused by ion binding include: (1) reduced hydration shell around the protein, (2) increased hydrogen bonding, (3) increased $\alpha$ helicity, particularly in the D and E-helix regions, and (4) reduced $\beta$-strand content between the CD and EF loops. We discuss each of these factors in order of importance based on their effects on the MM/GBSA scores, where applicable.

Our results summarized a variety of changes in binding loop dynamics, helical content, hydrogen bonding, and bundle packing upon ion binding to $\alpha \mathrm{PV}$ variants that were anticipated to impact the protein/solvent interface. Indeed, we found that the subtle conformational changes induced by ion binding manifested in reduced hydration of holo $\alpha \mathrm{PV}$ compared to apo and to a similar extent across all variants. This desolvation has been suggested to be a significant factor in driving ion binding in $\mathrm{Ca}^{2+}$-selective proteins [22, 21], which can be rationalized by the liberated solvent increasing the configurational entropy of the system. The displacement of loosely-bound waters in the CD and EF loops of the apo states upon binding $\mathrm{Ca}^{2+}$ represented a substantial portion of the total numbers of waters liberated in the holo state. It is unclear how much each liberated water contributed to the $\mathrm{Ca}^{2+}$ affinity, although one report suggested that a Glu to Asp 
mutation reduced $\mathrm{Ca}^{2+}$ affinity by five fold estimated based on $K_{D}^{\prime} / K_{D}=\exp (-\Delta \Delta G / K T)$ due to an extra coordinated water [17].

We attributed the remainder of the displaced waters to reorganization of the helical packing that apparently reduced the proteins' solvent accessible surface area. It has been speculated that the reduced affinity of CBPs for $\mathrm{Mg}^{2+}$ relative to $\mathrm{Ca}^{2+}$ can be partially attributed to the former ion's reduced capacity to displace waters [22], but our data showed no significant differences between the ions in terms of protein dehydration. While we were unable to determine the relative thermodynamic contribution of these displaced waters to the $\mathrm{MM} / \mathrm{GBSA}$-estimated scores we calculated, the greater number of waters displaced in the $\mathrm{S} 55 \mathrm{D} / \mathrm{E} 59 \mathrm{D} \alpha \mathrm{PV}$ and WT cases relative to D94S/G98E $\alpha \mathrm{PV}$ is consistent with their trends in stronger experimentally-measured binding affinities.

The dampened RMSF values and dehydration of holo-state proteins relative to apo states reported in our simulations implicate changes to the organization of the proteins' helix bundles. Unlike $\mathrm{Ca}^{2+}$ binding proteins such as calmodulin (CaM) that undergo significant conformational changes upon $\mathrm{Ca}^{2+}$ binding, the $\alpha \mathrm{PV}$ proteins exhibit comparatively more subtle changes. To highlight these subtle effects, we measured the net number of hydrogen bonds formed during the apo to holo state transition, as these bonds would be expected to yield larger free energy contributions than corresponding interactions between apolar residues. We found consistent increases in the number of intraprotein hydrogen bonds in the holo states of the S55D/E59D variant and the WT $\alpha \mathrm{PV}$ compared to $\Delta \alpha \mathrm{PV}$, which were correlated with the pattern of dehydration discussed in the previous section. Some of this increase was attributed to the extension of the D and E helices' $\alpha$-helicity, although this increase was generally similar across the variants. Increasing hydrogen bonds generally was observed in the holo versus apo states, with similar changes noted for $\mathrm{Ca}^{2+}$ and $\mathrm{Mg}^{2+}$. However, longer duration hydrogen bonds appear to be evident in the stronger binders of $\mathrm{Ca}^{2+}$, which may contribute to stabilization of the bound complex.

$\mathrm{Ca}^{2+}$ binding proteins commonly exert regulatory control through interfacing with target proteins. In many cases, such as for CaM and TnC, the protein-protein interface comprises the CBP's solvent-exposed hydrophobic patch and a coiled domain from the target peptide. The binding of the target peptide is often associated with an increase in the apparent $\mathrm{Ca}^{2+}$ affinity of the CBPs and increased thermostability [18, 62]. The CD and EF helix-loop-helix motifs in the parvalbumin class of CBPs bear high structural similarity to the globular domains of CaM and TnC. Uniquely, the AB domains in PV assume a position similar to that of the target-bound protein-protein interface in $\mathrm{CaM}$ and $\mathrm{TnC}$, where it binds its $\mathrm{CD}$ and EF domains through seven hydrogen bonds. In this regard, the AB domains in PV serve as an 'endogenous' peptide that confers thermostabilty and enhanced $\mathrm{Ca}^{2+}$ affinity. Indeed, experimental studies support that this $\mathrm{AB}$ domain is essential for stabilizing the apo-state $\alpha \mathrm{PV}$ into its folded form and protects the $\alpha \mathrm{PV}$ hydrophobic core from hydration, whereby $\mathrm{Ca}^{2+}$ affinity is enhanced [18]. Interestingly, a truncated PV variant that lacks the $\mathrm{AB}$ domain maintains the ability to bind $\mathrm{Ca}^{2+}$ albeit with significantly reduced affinity, whereas a similar truncation in the structurally-similar calbindin $9 \mathrm{~K}$ is incapable of binding the ion [62]. 
Our simulation results confirm that the intact $\alpha \mathrm{PV}$ has increased hydrogen bonding and reduced bundle mobility as evidenced by RMSF calculations, helicity, and dehydration upon $\mathrm{Ca}^{2+}$ binding relative to the truncated variant. The differences highlight the importance of the $\mathrm{AB}$ domains in defining $\alpha \mathrm{PV}^{\prime} \mathrm{s} \mathrm{Ca}^{2+}$ binding properties. Supporting these observations, in the holo state there is a loss of favorable reorganization score of the CDEF domain in the truncated variant compared to the intact one (Table 1). Despite this loss of stability, the $\Delta \alpha \mathrm{PV}$ holo state remains folded in the presence of $\mathrm{Ca}^{2+}$ as supported by the Thepaut et al experiments [18]. Nonetheless, even though the $\Delta \alpha \mathrm{PV}$ holo structure is folded, it presents high RMSF values that suggest instability within the helix bundle relative to the intact $\alpha \mathrm{PVvariants}$, in addition to reduced D-helicity and enhanced $\beta$-sheet character that may impair $\mathrm{Ca}^{2+}$ binding. While we were unable to predict the unfolded apo state indicated by Thepaut et al, our simulations suggest that the $\Delta \alpha \mathrm{PV}$ apo structures were unfolding and highly labile relative to the intact $\alpha \mathrm{PV}$ structure. From this perspective, the presence of the $\mathrm{AB}$ domains may amortize the thermodynamic cost of folding the protein upon binding $\mathrm{Ca}^{2+}$, which would otherwise reduce the $\alpha \mathrm{PV}$ apparent $\mathrm{Ca}^{2+}$ affinity. In support of this interpretation, lysine-to-asparagine mutations of the AB domain sites A8 and H26 in PV constructs modeled after Antartic fish were found to increase $\mathrm{Ca}^{2+}$ binding affinity and was attributed to forming additional hydrogen bonds within the $\mathrm{AB}$ or between $\mathrm{AB}$ and $\mathrm{CDEF}$ domains [63]. Overall, our observations highlight the importance of $\mathrm{AB} / \mathrm{CDEF}$ domain interactions for maintaining high $\mathrm{Ca}^{2+}$ binding affinity.

As with many $\mathrm{Ca}^{2+}$ binding proteins, $\alpha \mathrm{PV}$ is nearly entirely composed of $\alpha$-helices that undergo displacements and changes in folding upon binding by $\mathrm{Ca}^{2+}[64,65,66,22]$. We observed a consistent trend in our simulations of increased $\alpha$-helicity in the ion-bound holo states relative to apo, with greater gains demonstrated for $\mathrm{Ca}^{2+}$ relative to $\mathrm{Mg}^{2+}$. This observation is consistent with observations from Laberge et al, who through ultraviolet circular dichroism spectra and picosecond MD simulations of cod PV [67] showed that removal of $\mathrm{Ca}^{2+}$ reduced $\alpha$-helical content. Our simulations indicate that the majority of the gain in $\alpha$-helical character stems from refolding of the N-terminal end of the D helix. At these termini, glutamic acids at the 59th and 62nd sequence positions (E59D for S55D/E59D $\alpha \mathrm{PV}$ ) are reoriented by $\mathrm{Ca}^{2+}$ as the ion binds the carboxylic acid side chains. This process increases the helicity of residues E/D59 through G64 to $90 \%$. In contrast, we observed increase in helicity in the C-terminal end of the $\mathrm{E}$ helix, particularly for the residues T84-D90.

Interestingly, several observations are notable: firstly, $\mathrm{Ca}^{2+}$-driven changes in D-helix $\alpha$ character were limited to the N-terminus up to G64. Glycine is frequently found to disrupt or break $\alpha$-helices [68, 50], thus we speculate that varying the relative position of this residue within the D-helix could modulate $\mathrm{Ca}^{2+}$ affinity (see Section S8.4 for further discussion); second, our $\Delta \alpha \mathrm{PV}$ simulations reflected a very low (55\%) degree of helicity formed in the D-helix N-terminus, which suggests the $\mathrm{AB}$ domains likely play an important role in stabilizing the $\mathrm{Ca}^{2+}$ binding loop, which in turn may increase its affinity for $\mathrm{Ca}^{2+}$; similarly, weaker binding $\alpha \mathrm{PV}$ variants tend to have less helical character in their apo states. Lastly, the D-helix N-terminus has a lesser degree of $\alpha$-helicity in the $\mathrm{Mg}^{2+}$-bound state (65\%). It has been speculated that this difference may be 
the consequence of the 12th position glutamic acid (E62) promoting bidentate oxygen coordination for $\mathrm{Ca}^{2+}$ relative to monodentate for $\mathrm{Mg}^{2+}[22,37]$. No such differences were noted in the E-helix between the $\mathrm{Ca}^{2+}$ and $\mathrm{Mg}^{2+}$ ions. Altogether, our data indicate that increasing the $\alpha$-helicity particularly in the N-terminal region of helices $\mathrm{D}$ and $\mathrm{E}$, may play an integral role in high affinity $\mathrm{Ca}^{2+}$ binding and selectivity against $\mathrm{Mg}^{2+}$.

A hallmark of $\mathrm{Ca}^{2+}$ binding proteins is the pairing of two $\mathrm{Ca}^{2+}$-binding $\mathrm{EF}$ hand domains via a $\beta$-sheet, which has been coined as the "EF-hand $\beta$-scaffold (EFBS)" [22]. These $\beta$-sheets are believed to contribute to the $\mathrm{Ca}^{2+}$ binding mechanism and cooperativity for some CBPs [22]. Our studies support the notion that $\beta$-sheet character is reduced upon $\mathrm{Ca}^{2+}$ binding and to a lesser extent by $\mathrm{Mg}^{2+}$. Among the intact $\alpha \mathrm{PV}$ variants, the reduction correlates with $\mathrm{Ca}^{2+}$ affinity and analogously, the low $\mathrm{Ca}^{2+}$-affinity $\Delta \alpha \mathrm{PV}$ variant presents the highest degree of $\beta$-sheet character when bound to $\mathrm{Ca}^{2+}$. It is evident from published structural data and simulations that the reduction in $\beta$-strand content occurs as the residues contributed by each site (F57-E59 and K96-G98) are drawn away from the $\beta$-sheet interface by their respective coordinated ions. The largest displacements arise from E59 and K96 as they are separated from one another by S55 and D94, respectively, through the formation of hydrogen bonds (Fig. S9). Our simulation data indicate that the coordination geometries and extent of $\beta$-sheet character were similar among the holo variants, whereas the apo state $\beta$-sheet content increased for variants with reduced $\mathrm{Ca}^{2+}$ affinity. Hence, the reduced binding affinity for $\mathrm{Ca}^{2+}$ in those variants likely reflects the thermodynamic cost of disrupting a greater number of hydrogen bonds participating in the $\beta$-sheet interface. Interestingly, it has been reported that the coupling between the two binding sites through hydrogen bonds and dipole-dipole interactions improved $\mathrm{Ca}^{2+}$ affinity to the CBPs through positive cooperativity [20], although the lack of cooperativity in $\alpha \mathrm{PV}$ may give rise to this contradictory trend in our data. Overall, our simulation data suggest that mutations that enhance $\beta$-sheet character in the apo state appear to offset the free energy gain upon $\mathrm{Ca}^{2+}$ binding.

\subsection{Molecular determinants of $\mathrm{Ca}^{2+}$ versus $\mathrm{Mg}^{2+}$}

$\alpha \mathrm{PV}$ selects for $\mathrm{Ca}^{2+}$ over the smaller $\mathrm{Mg}^{2+}$ ion that is roughly 10000-fold more concentrated within cells [22]. The experimentally-determined affinity difference of $\approx 10 \mathrm{kcal} / \mathrm{mol}$ in large part can be rationalized by the considerably higher desolvation cost for $\mathrm{Mg}^{2+}$ relative to $\mathrm{Ca}^{2+}$ and to a lesser extent by the reduced number of protein oxygens bound by $\mathrm{Mg}^{2+}$ [22], as was confirmed by our MSA calculations. Additional factors, however, though can further tune the observed affinity differences for these ions, including the energetic cost of distorting the EF-hand to accommodate the smaller $\mathrm{Mg}^{2+}$ ion over $\mathrm{Ca}^{2+}$ [37], polarizability [69, 70] and many-body polarization effects [26], which depend on the number and the arrangement of charged residues. Regardless of the origins of the affinity differences, our simulations reveal distinct differences between the holo states of $\mathrm{Mg}^{2+}$-bound $\alpha \mathrm{PV}$ structures relative to those bound with $\mathrm{Ca}^{2+}$. These differences include reduced $\alpha$-helicity, especially within the D-helix N-terminal region, and less significant disruption of the $\beta$-sheets bridging the EF-hand loops. These secondary structure differences are mirrored by the MM/GBSA scores 
from these corresponding regions in the $\alpha \mathrm{PV}$ proteins. Similarly, it could be argued that those differences may have significant impact on proteins such as TnC, for which modest differences in EF-hand structures when bound to $\mathrm{Mg}^{2+}$ versus $\mathrm{Ca}^{2+}$ dictate the exposure of hydrophobic surfaces necessary for binding peptide substrates. In contrast, the presence of the $\mathrm{AB}$ domains in $\alpha \mathrm{PV}$ most likely masks such structural differences that would arise for $\mathrm{Ca}^{2+}$-bound states relative to $\mathrm{Mg}^{2+}$ bound. Instead, the $\mathrm{AB}$ domains appears to lock $\alpha \mathrm{PV}$ into an open-like state resembling that of $\mathrm{TnC}$ even in the absence of $\mathrm{Ca}^{2+}$, which thereby facilitates binding of other competing cations such as $\mathrm{Mg}^{2+}$. This interpretation is supported by studies from Thepaut et al that indicated $\mathrm{Mg}^{2+}$ was unable to fold the $\Delta \alpha \mathrm{PV}$ variant that lacked the $\mathrm{AB}$ helices. For this reason, the prominence of parvalbumin proteins serving as delayed $\mathrm{Ca}^{2+}$ buffers in skeletal and cardiac muscle [71] likely stems from its $\mathrm{AB}$ domain facilitating $\mathrm{Mg}^{2+}$ binding and thereby competing with $\mathrm{Ca}^{2+}$ binding.

\section{Conclusions}

In this report, we investigated several structural features in $\alpha \mathrm{PV}$ that factor into the proteins' affinity for $\mathrm{Ca}^{2+}$ and its selectivity over $\mathrm{Mg}^{2+}$. Our approach utilized molecular dynamics simulations of the wild-type $\alpha \mathrm{PV}$ ion-free (apo) and $\mathrm{Ca}^{2+} / \mathrm{Mg}^{2+}$-bound (holo) states, in addition to simulations of $\alpha \mathrm{PV}$ variants with increased or reduced $\mathrm{Ca}^{2+}$ affinity relative to the WT. We demonstrate that the MM/GBSA endpoint method (when applied to the protein reorganization score) and MSA theory are effective tools for qualitatively rank ordering the intact $\alpha \mathrm{PV}$ variants (WT, S55D/E59D $\alpha \mathrm{PVand}$ D94S/G98E $\alpha \mathrm{PV}$ ) by their experimentally determined affinities. We used scores from these analyses to then implicate structural changes that rationalize the variants' ion binding affinities. We find that simple metrics such as the number of coordinating oxygens about a bound ion or changes in the binding sites' net charge are insufficient to completely rationalize observed changes in affinity. Rather, concurrent effects on the protein's secondary structure composition, solvation properties, and hydrogen bonding networks throughout its globular domain are involved and must be carefully analyzed to understand the effects of mutations and evolutionary differences between PV isoforms. Clearly, rationalizing affinity in $\mathrm{Ca}^{2+}$ binding proteins including PV therefore necessitates not only knowledge of the proteins' primary sequence and corresponding structures, but also how those structures impact the thermodynamics of the $\mathrm{Ca}^{2+}$ free and bound states. In many cases, this may require looking beyond direct effects of changing the charge or $\mathrm{Ca}^{2+}$ coordination within the EF hands [72]. This firmly indicates the value of molecular simulations in probing residue-specific contributions to protein stability and $\mathrm{Ca}^{2+}$ binding in $\alpha \mathrm{PV}$. Thus, it is apparent from our data that point mutations can modulate affinity using diverse mechanisms.

An important outcome of our study are potential mechanisms that can be manipulated through mutagenesis to tune $\mathrm{Ca}^{2+}$ affinity. As an example, introducing mutants that destabilize the $\beta$-strands linking the CD and $\mathrm{EF}$ hand loops in the apo state may amortize their reorganization when binding $\mathrm{Ca}^{2+}$. Additional strategies could include increasing the apo state $\alpha$-helicity by replacing the helix-breaking G64 with a small hydrophobic residue like alanine (G64A) that would restore normal helicity, introducing additional hydrogen-bonding polar 
residues to bridge the $\mathrm{AB}$ and $\mathrm{CDEF}$ interface and thereby tighten $\mathrm{AB} / \mathrm{CDEF}$ binding, as well enriching the $\mathrm{CD}$ and EF loops with glycine to further increase their mobility.

\section{Materials and methods}

\subsection{Protein systems}

$\mathrm{Ca}^{2+}$ bound rat $\alpha \mathrm{PV}$ X-ray crystal structures (PDBs: 1RWY [40], 1XVJ [16], 1S3P [15], and 1G33 [18]) were used for the MD simulations. Apo simulations were set up by removing ions from the holo crystal structures. Because crystal structures of $\mathrm{Mg}^{2+}$ bound and apo $\alpha \mathrm{PV}$ protein are not yet available, $\mathrm{Ca}^{2+}$ was replaced with $\mathrm{Mg}^{2+}$ for the $\mathrm{Mg}^{2+}$ bound simulations. System preparation was performed with tleap from Amber 16 [73].

\subsection{Classical MD}

All systems were solvated in TIP3P [74] water box with $20 \AA$ margin and neutralized with $0.15 \mathrm{M} \mathrm{KCl}$. Each system has about 14,000 waters, 82 neutralizing ions, for a total of $\approx 44,000$ atoms. The systems were parameterized with the amber ff12SB force field [75] with $\mathrm{Ca}^{2+}$ and $\mathrm{Mg}^{2+}$ parameters were adapted from Li-Merz [29]. The system was subjected to 6000 steps minimization with protein atoms being fixed during the first 1000 steps. The system was then heated under NVT ensemble to $300 \mathrm{~K}$ over $0.1 \mathrm{~ns}$ using the weak-coupling algorithm [76]. Constraints were introduced on protein atoms with force constant as 500 $\mathrm{kcal} / \mathrm{mol} . \AA^{2}$. The heated system was equilibrated for $1 \mathrm{~ns}$ under the NPT ensemble without constraints on the protein. After equilibrium, each system was simulated in triplicate for $\approx 1 \mu$ s under the NPT ensemble at $300 \mathrm{~K}$ temperature. A 2 fs time step was used with the non-bonded cutoff set as $10 \AA$ and the electrostatic interactions were treated the with Particle Mesh Ewald (PME) method [77]. The SHAKE algorithm was used [78]. The simulation data of the production run were saved for every $20 \mathrm{ps}$. The minimization and equilibration were performed using the PMEMD module while the production runs were performed using the GPU-accelerated PEMD.CUDA module of AMBER16 [73].

\subsection{Analyses}

Two snapshots for each ns of trajectory were considered for the analysis $(\approx 2000$ data points for each simulation trajectory $(\approx 1 \mu \mathrm{s})$ and a total of 72000 data points for all systems combined (12 systems $\times 3$ trials $\times 2000$ data points $/ 1 \mu \mathrm{s}) \approx 72000$ data points). Almost all analyses were conducted with VMD [79] and its associated plugins $[79,80]$, except for the radial distribution functions. For ion bound systems, the radial distribution of chelating oxygens about the cation (either $\mathrm{Ca}^{2+}$ or $\mathrm{Mg}^{2+}$ ) was obtained by the radial command of CPPTRAJ [81]. For calculating hydrogen bonds, the cut-off distance and angle used were $3.5 \AA$ and $30^{\circ}$ respectively. RMSDs and RMSFs were calculated by superposing each trajectory frame with the respective x-ray crystal structure. The MMPBSA.py script of AMBER16 was used for the MM/GBSA calculations. The ionic 
strength for the MM/GBSA calculation was set as $0.15 \mathrm{M}$ with the generalized Born model option set as igb $=5$. The entropy was neglected during the MM/GBSA calculations. The entire MD trajectories were used for the MM/GBSA calculations. The total score/reorganization score of each system was calculated as the sum of all five individual components of the MM/GBSA method including electrostatic, van der Waals, internal, polar and non-polar solvation, respectively.

\subsection{MSA analyses}

The mean field approximation theory (MSA) self-consistently solves for the optimal distribution of ions comprising an electrolyte solution within a protein binding domain, or 'filter', consisting of mobile oxygens. To do this, the model utilizes a Hamiltonian reflecting the free energy of packing finite-sized ions as hard spheres and electrolyte-shielded electrostatic energies in a fixed volume of oxygens that cannot exchange with the surrounding medium [46, 82]. This method includes the thermodynamics of solvent ions simultaneously competing with the binding site, the desolvation energies of those ions, as well as the finite sizes of ions and oxygens in the binding domain. Ion distributions, $\rho$, are predicted from the chemical potential, $\mu$, which arises from the balance between (un)favorable electrostatic interactions and entropic hard-sphere interactions $[83]$.

All ions, namely $\mathrm{Ca}^{2+}, \mathrm{Mg}^{2+}, \mathrm{K}^{+}$and $\mathrm{Cl}^{-}$can readily repartition in order to minimize the gradient in the chemical potential:

$$
\mu_{i}^{e x}=\mu_{i}^{E S}+\mu_{i}^{H S}
$$

Further details of the forms of $\mu_{i}^{E S}$ and $\mu_{i}^{H S}$ are provided in the supplementary material of [38]. At steady state, for each ion species, the following equilibration is reached:

$$
k_{B} T \ln \left(\rho_{i}\right)=k_{B} T \ln \left(\rho_{0, i}\right)+\mu_{0, i}^{e x}-z_{i} e_{0} \Psi-\mu_{i}^{e x}+G_{\text {solv }}
$$

where $\rho_{i}$ is the density of the i-th ion type in the binding site, $\rho_{0, i}$ is the density of the i-th ion in the bath (assumed to remain constant), $\mu_{i}^{e x}$ is the excess chemical potential of the $\mathrm{i}$-th ion in the binding site, $\mu_{0, i}^{e x}$ is the excess chemical potential of the i-th ion in the bath (also constant), $z_{i}$ is the integer charge of the i-th ion species, $\Psi$ is the Donnan potential for the binding site, $G_{\text {solv }}$ is the solvation energy of the i-th ion.

\section{Acknowledgements}

Research reported in this publication, release was supported by the Maximizing Investigators' Research Award (MIRA) (R35) from the National Institute of General Medical Sciences (NIGMS) of the National Institutes of Health (NIH) under grant number R35GM124977. PKH thanks Caitlin Scott, Charles Adeniran and Andrew Mondragon for prototyping simulation conditions for this project. We also thank XSEDE for providing resources for conducting the simulations [84]. 


\section{S8 Supplement}

\section{S8.1 Figures}

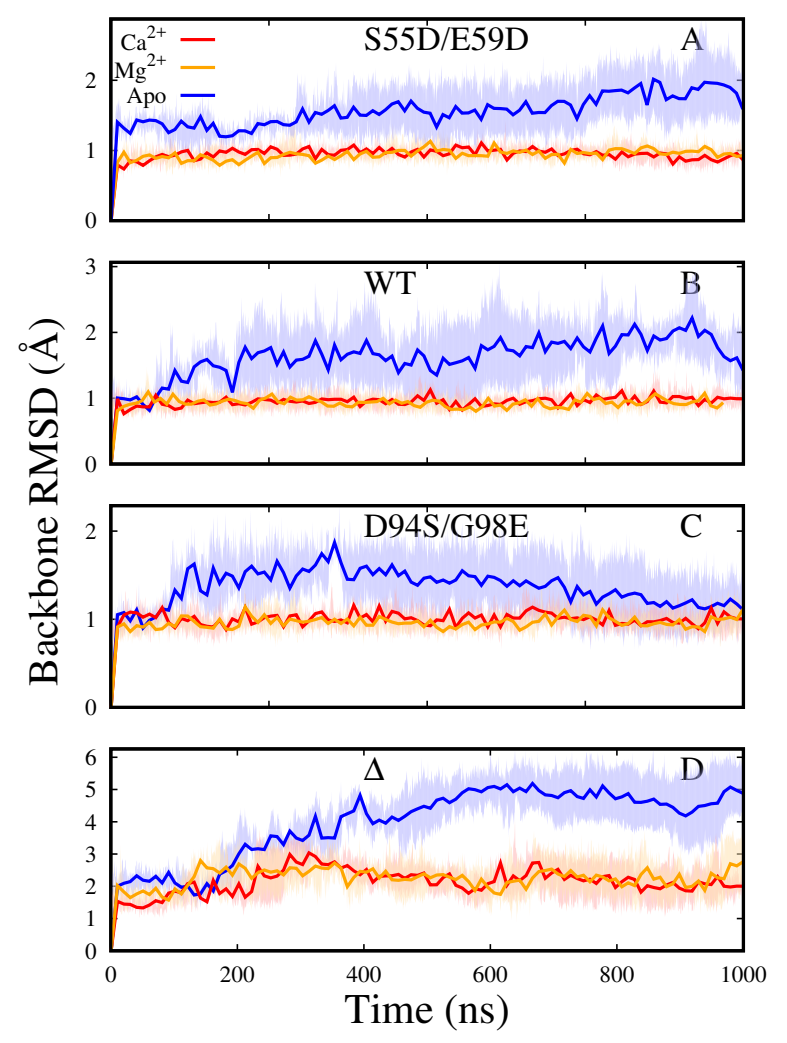

Figure S1: Moving average of the backbone RMSD for S55D/E59D $\alpha \mathrm{PV}, \alpha \mathrm{PV}, \mathrm{D} 94 \mathrm{~S} / \mathrm{G} 98 \mathrm{E} \alpha \mathrm{PV}$ and $\Delta \alpha \mathrm{PV}$ protein. Apo, $\mathrm{Ca}^{2+}$, and $\mathrm{Mg}^{2+}$ bound systems are colored blue, red and orange respectively. Dark lines represent the average and shaded regions represent the standard deviation of three MD runs. 

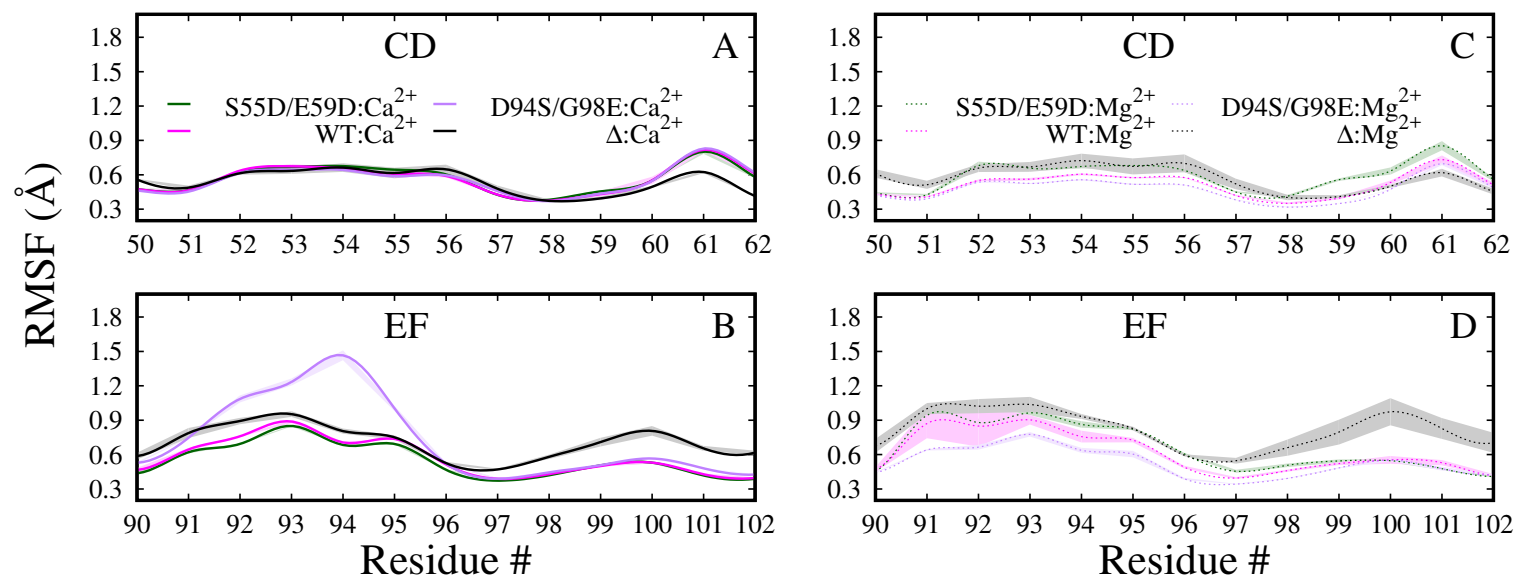

Figure S2: $\quad$ Root mean square fluctuation (RMSF) of the CD (A, C) and EF (B, D) sites of holo systems. $\mathrm{Ca}^{2+}$ bound sytems are shown in panels $\mathrm{A}$ and $\mathrm{B}$ and $\mathrm{Mg}^{2+}$ bound in the panels $\mathrm{C}$ and D. Mean and standard deviation of three MD trials are shown.

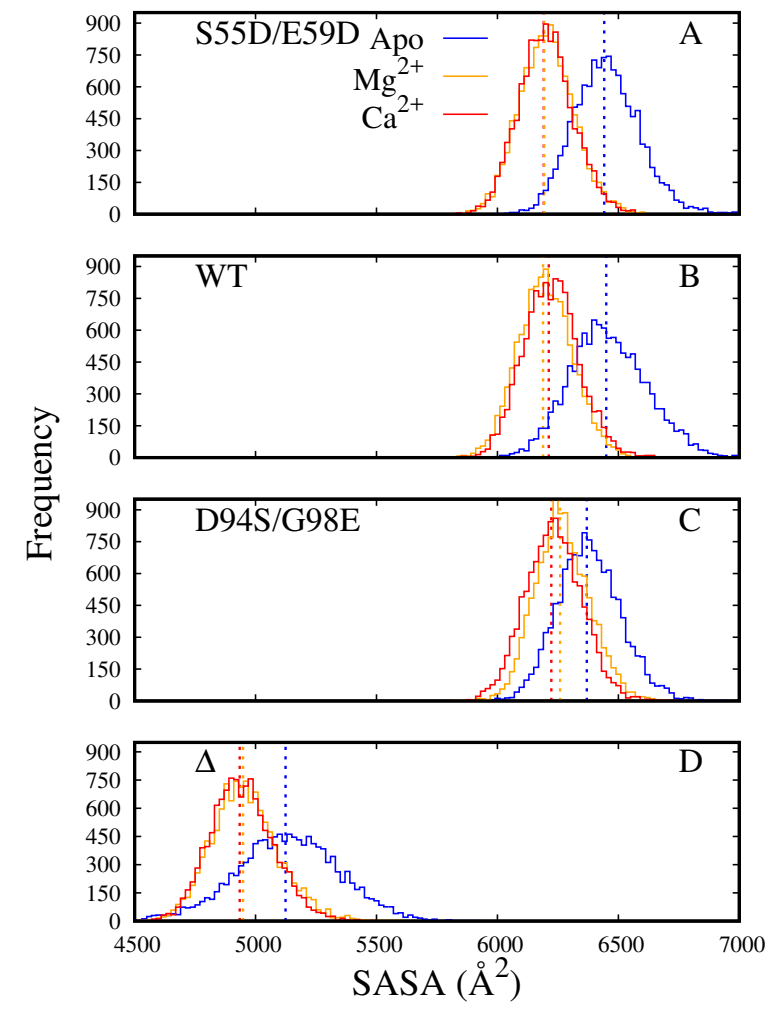

Figure S3: Distributions of solvent accessible surface area (SASA) of entire protein. Apo, Ca ${ }^{2+}$, and $\mathrm{Mg}^{2+}$ bound systems are colored blue, red and orange respectively. Averages of all three trials for each $\alpha \mathrm{PV}$ variant are represented by dashed vertical lines.. 


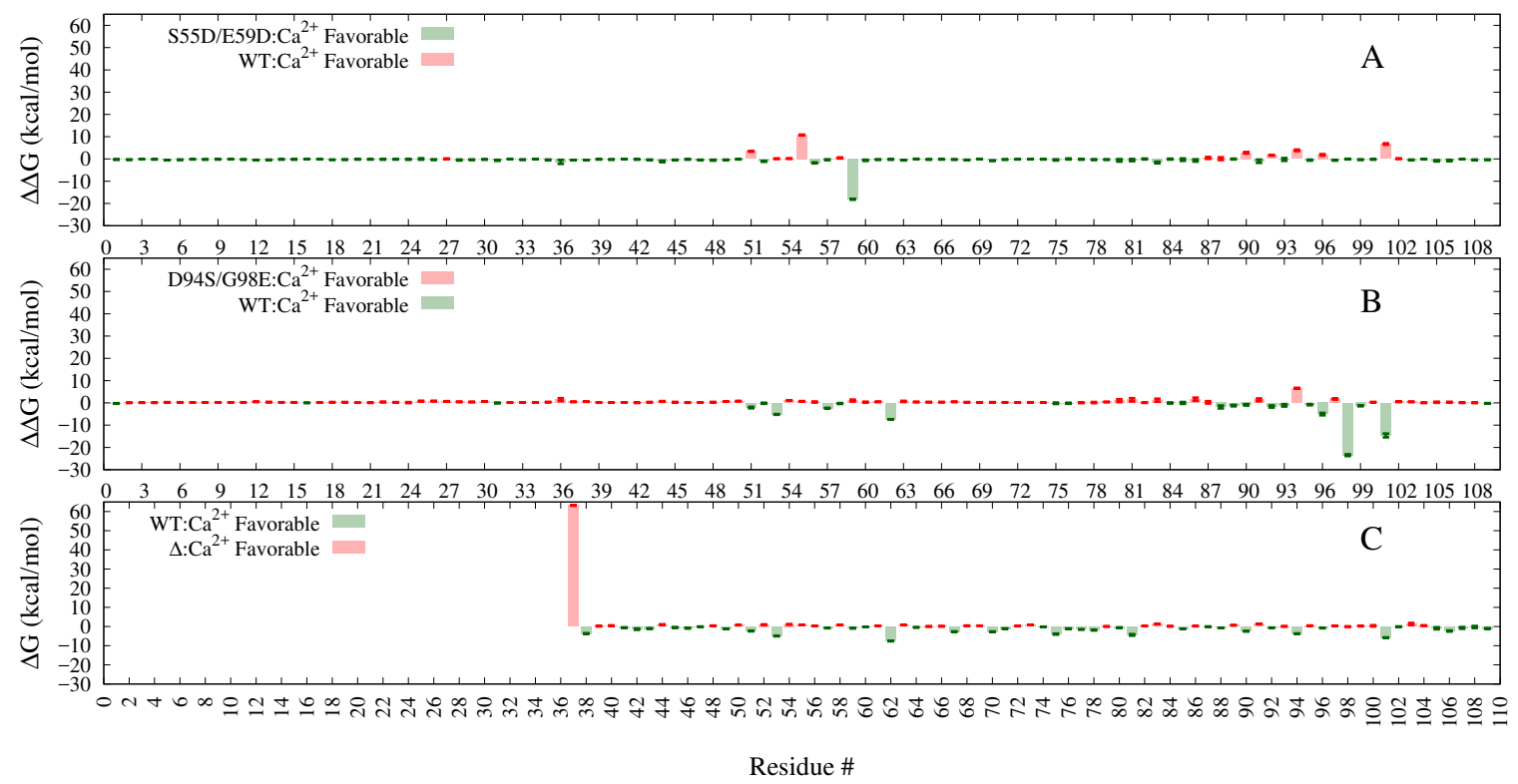

Figure S4: Residue reorganization energies of all $\mathrm{Ca}^{2+}$ bound systems calculated using MM/GBSA. The MD predicted $\Delta \alpha \mathrm{PV}$ apo does not unfold as expected from experiment [18], hence only free energies of holo state are compared. See Table S3 and Table S4 for summations of structurally-significant regions. Mean and sem of each residue estimated from three MD trials are shown .

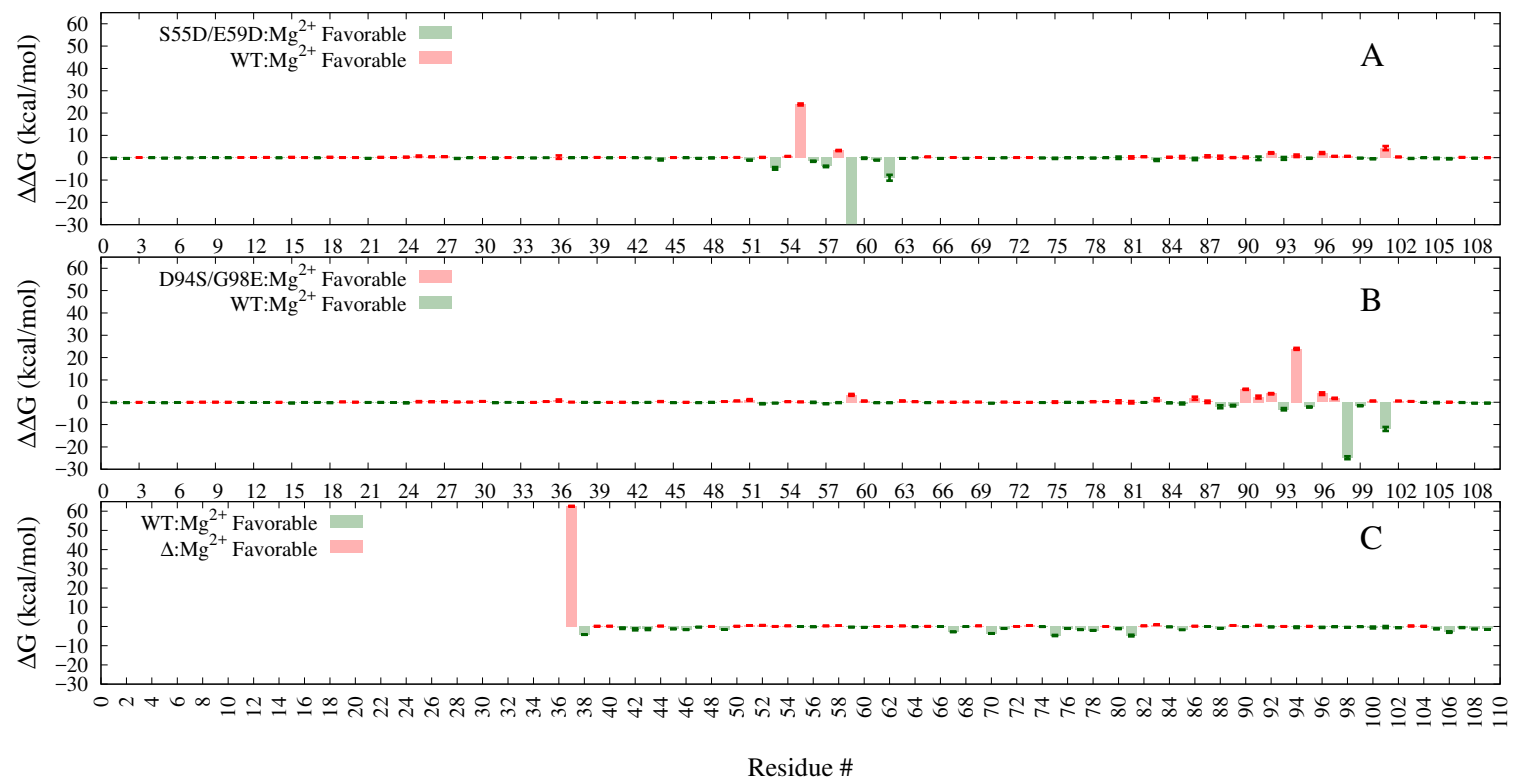

Figure S5: Comparison of MM/GBSA residue reorganization energies of all $\mathrm{Mg}^{2+}$ bound systems. Mean and sem of each residue estimated from three MD trials are shown. 

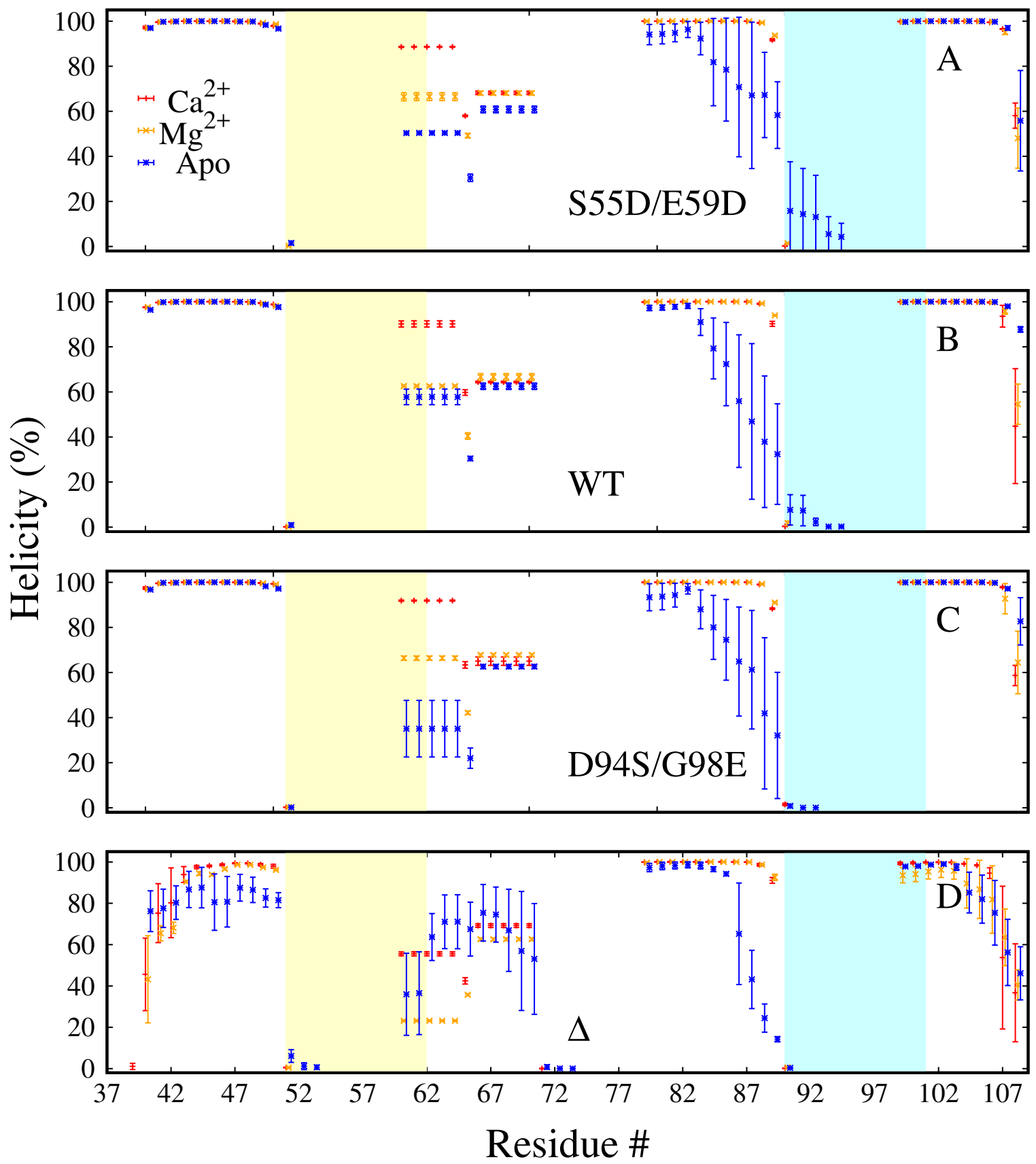

Figure S6: $\quad \alpha$-helical content versus residue number. Apo, $\mathrm{Ca}^{2+}$ and $\mathrm{Mg}^{2+}$ bound systems are colored blue, red and orange respectively. CD and EF site loop domains are colored yellow and blue respectively. Error bars represent standard deviation estimated from three MD runs. 

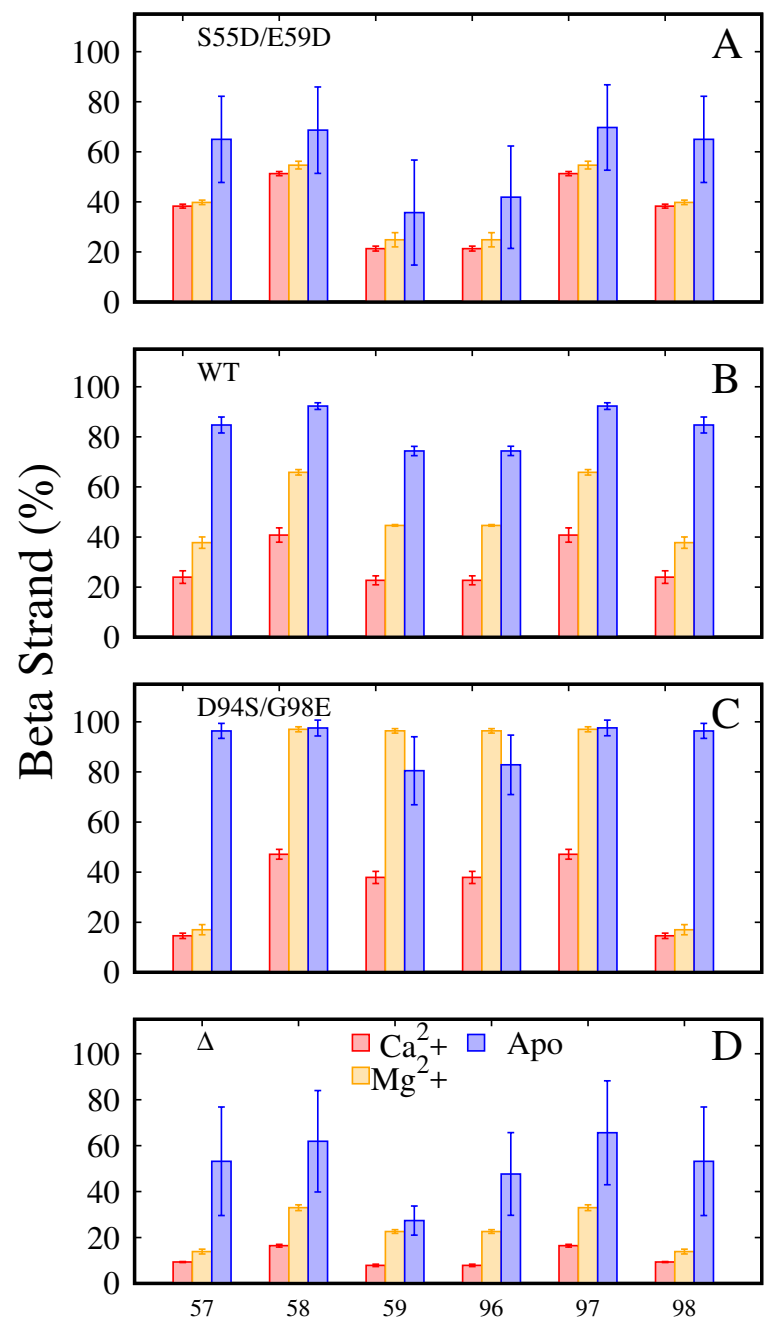

Residue \#

Figure S7: $\quad \beta$-strand (A-D) of the six residues (F57-E59 and K96-G98) that form the $\beta$ - $\beta$ bridge coupling the two ion binding sites. Apo, $\mathrm{Ca}^{2+}$ and $\mathrm{Mg}^{2+}$ bound systems are colored blue, red and orange respectively. Error bars represent standard deviation. 


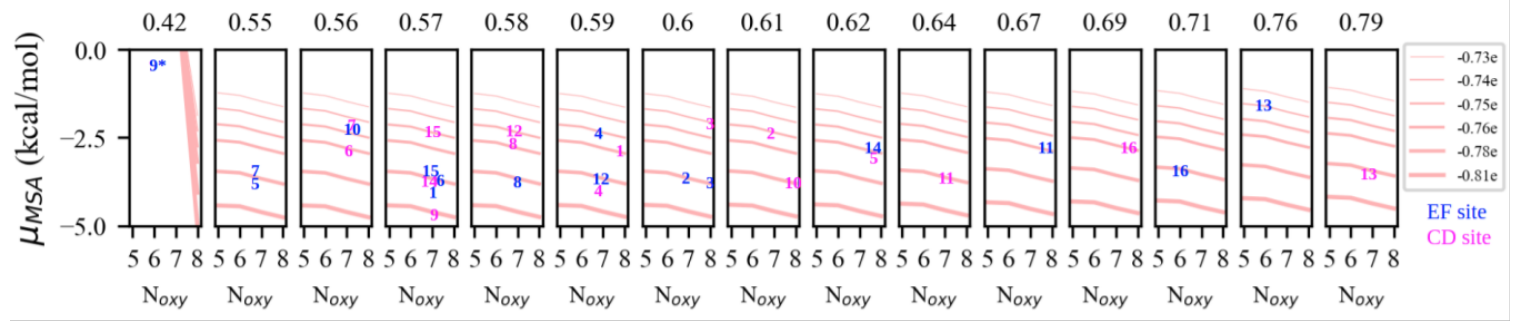

Figure S8: Mean spherical approximation (MSA) calculated $\mathrm{Ca}^{2+}$ potentials using binding site information from PDB structures of PV isoforms that have above $50 \%$ identity with $\alpha \mathrm{PV}$ (PDB 1RWY). The identities were calculated via the protein BLAST tool from the NCBI website using the amino acid sequence of PDB 1RWY as input. These PV isoforms are: 1-whiting PV (PDB 1A75), 2- $\beta$ PV (PDB 1B8R), 3- $\beta$ PV (PDB 1B9A), 4-silver hake PV (PDB 1BU3), 5-carp PV (PDB 1CDP), 6-oncomodulin (PDB 10MD), 7-pike PV (PDB 1PAL), 8-pike PV (PDB 1PVA), 9-gadus PV (PDB 2MBX), 10-rat $\alpha \mathrm{PV}$ (PDB 3F45), 11-gallus $\beta \mathrm{PV}$ (PDB 3FS7), 12-leopard shark PV (PDB 5PAL), 13-pacific mackerel PV (PDB 5XND), 14-mustelus griseus PV (PDB 5ZGM), 15-mustelus griseus PV (PDB 5ZGM) and 16- $\alpha$ PV(PDB 1RK9). *The data for 2MBX $\mathrm{EF}$ site is not included as the cation-oxygen distance is suspiciously small. 

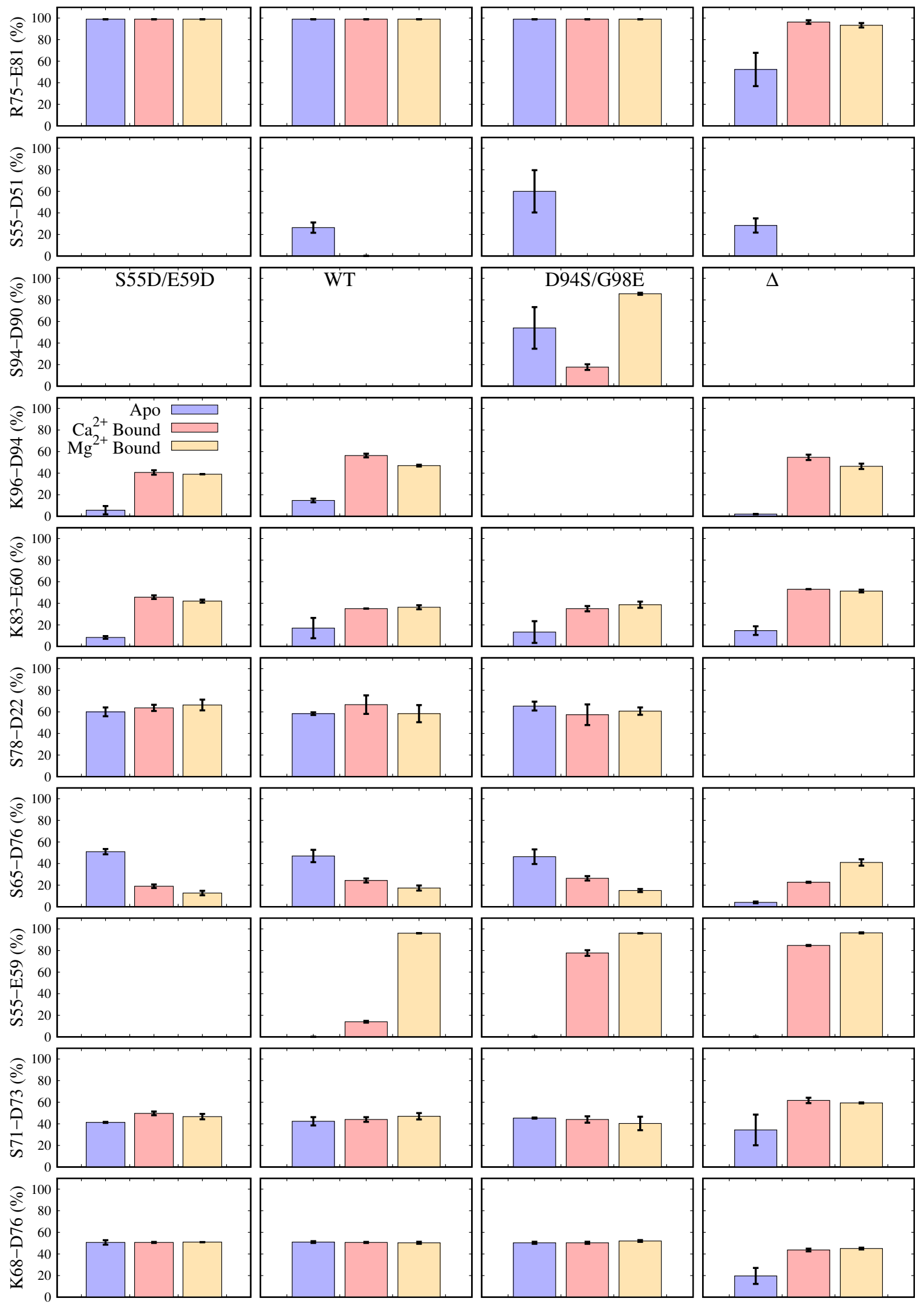

Figure S9: Comparison of specific hydrogen bonds (sidechain-sidechain). Columns 1, 2, 3 and 4 represent

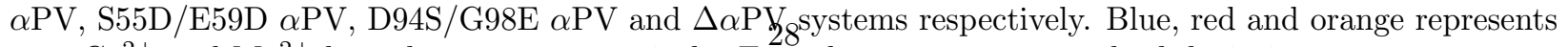
apo, $\mathrm{Ca}^{2+}$ and $\mathrm{Mg}^{2+}$ bound systems respectively. Error bars represent standard deviation. 


\section{S8.2 Tables}

Table S1: Estimated MM/GBSA scores and experimental binding affinities.

\begin{tabular}{l|cccc}
\hline System & $\Delta \mathbf{G}_{\text {Total }}^{\text {Calc }}$ & $\Delta \Delta \mathbf{G}_{\text {Total }}^{\text {Calc }}$ & $\Delta \mathbf{G}^{\text {Expt }}$ & $\Delta \Delta \mathbf{G}^{\text {Expt }}$ \\
\hline S55D/E59D $\alpha \mathrm{PV}: \mathrm{Ca}^{2+}$ & $-143.44 \pm 1.75$ & $-58.59 \pm 2.16$ & $(-24.0 \pm 0.21)[15,45]$ & $-2.0 \pm 0.22$ \\
$\alpha \mathrm{PV}: \mathrm{Ca}^{2+}$ & $-84.85 \pm 1.27$ & $0.00 \pm 1.79$ & $-22.0[15,16]$ & $0.0 \pm 0.08$ \\
D94S/G98E $\alpha \mathrm{PV}: \mathrm{Ca}^{2+}$ & $-141.02 \pm 1.92$ & $-56.18 \pm 2.30$ & $-18.2 \pm 0.21[16]$ & $3.8 \pm 0.22$ \\
$\Delta \alpha \mathrm{PV}: \mathrm{Ca}^{2+}$ & $-154.26 \pm 1.20$ & $-69.41 \pm 3.06$ & $-12.6[18]$ & 9.5 \\
\hline
\end{tabular}

$\Delta \overline{\mathrm{G}_{\text {Total }}^{\text {Calc }}}$ for each system is calculated as the difference of the of holo and apo component scores. Mean and sem of all three MD trials of each system are shown. $\Delta \Delta \mathrm{G}_{\text {Total }}^{\text {Calc }}$ is calculated relative score and $\Delta \Delta \mathrm{G}^{E x p t}$ is experimental relative free energy, and are calculated as the difference of score/free energies of the variants with the WT. Experimental values shown in parenthesis are at $5^{\circ} \mathrm{C}$ and the rest at $25^{\circ} \mathrm{C}$. All energies are in $\mathrm{kcal} / \mathrm{mol}$

Table S2: MM/GBSA reorganization energies of CD and EF site residues.

\begin{tabular}{|c|c|c|c|c|c|c|c|}
\hline System & $\mathrm{G}_{R E}^{\text {Calc }}(\mathrm{CD})$ & $\Delta \mathrm{G}_{R E}^{\text {Calc }}(\mathrm{CD})$ & $\Delta \Delta \mathrm{G}_{R E}^{\text {Calc }}(\mathrm{CD})$ & $\mathrm{G}_{R E}^{\text {Calc }}(\mathrm{EF})$ & $\Delta \mathrm{G}_{R E}^{\text {Calc }}(\mathrm{EF})$ & $\Delta \Delta \mathrm{G}_{R E}^{\text {Calc }}(\mathrm{EF})$ & $\Delta \Delta \mathrm{G}_{R E}^{\text {Calc }}($ Total $)$ \\
\hline S55D/E59D $\alpha \mathrm{PV}_{\mathrm{Ca}^{2+}}$ & & $59.13 \pm 1.23$ & $-7.83 \pm 1.33$ & & $57.66 \pm 0.17$ & $13.96 \pm 0.90$ & $6.13 \pm 1.61$ \\
\hline$\alpha \mathrm{PV}$ & $(-265.86 \pm 0.06)$ & $66.96 \pm 0.52$ & $0.00 \pm 0.74$ & $(-248.05 \pm 0.09)$ & $43.70 \pm 0.88$ & $0.00 \pm 1.25$ & $0.00 \pm 1.46$ \\
\hline D94S/G98E o & & $79.60 \pm 1.01$ & $12.64 \pm 1.14$ & & $81.94 \pm 1.87$ & $38.24 \pm 2.07$ & $50.8 \pm 2.37$ \\
\hline$\Delta \alpha \mathrm{PV}^{\prime} \mathrm{Ca}^{2+}$ & $(-254.13 \pm 0.04)$ & $\begin{array}{c}79.18 \pm 1.32 \\
(11.73 \pm 0.07)\end{array}$ & $12.22 \pm 1.42$ & $(-237.57 \pm 0.37)$ & $\begin{array}{c}55.90 \pm 1.08 \\
(10.48 \pm 0.38)\end{array}$ & $12.20 \pm 1.39$ & $24.42 \pm 1.99$ \\
\hline S55D/E59D $\alpha \mathrm{PV}: \mathrm{Mg}^{2+}$ & & $117.01 \pm 2.70$ & $-25.26 \pm 2.76$ & & $116.75 \pm 1.13$ & $9.59 \pm 1.65$ & $-15.67 \pm 3.22$ \\
\hline$\alpha \mathrm{PV}: \mathrm{Mg}^{2+}$ & $(-190.54 \pm 0.21)$ & $142.28 \pm 0.56$ & $0.00 \pm 0.80$ & $(-184.60 \pm 0.82)$ & $107.15 \pm 1.20$ & $0.00 \pm 1.70$ & -188 \\
\hline D94S/G98E $\alpha \mathrm{PV}: \mathrm{Mg}^{2+}$ & & $138.79 \pm 1.01$ & $-3.49 \pm 1.16$ & & $108.21 \pm 1.67$ & $1.05 \pm 2.06$ & $-2.43 \pm 2.37$ \\
\hline$\Delta \alpha \mathrm{PV}: \mathrm{Mg}^{2+}$ & $(-192.21 \pm 0.04)$ & $\begin{array}{c}141.10 \pm 1.32 \\
(-1.67 \pm 0.21)\end{array}$ & $-1.17 \pm 1.44$ & $(-182.21 \pm 1.16)$ & $\begin{array}{c}111.26 \pm 1.53 \\
(2.31 \pm 1.42)\end{array}$ & $4.10 \pm 1.95$ & $2.92 \pm 2.43$ \\
\hline
\end{tabular}

Estimates just considering the holo states $\left(\mathrm{G}_{R E}^{\text {Calc }}\right)$ are shown in parenthesis. For the CD site residues 50 to 62 and for the EF site residues 90 to 102 are considered. All the terms are explained in Table 1 in detail. All energies are in kcal/mol. Error bars represent sem.

Table S3: MM/GBSA reorganization energies of $\beta$-sheet residues.

\begin{tabular}{l|ccc}
\hline System & $\mathbf{G}_{R E}^{\text {Calc }}(\beta)$ & $\Delta \mathbf{G}_{R E}^{\text {Calc }}(\beta)$ & $\Delta \Delta \mathbf{G}_{R E}^{\text {Calc }}(\beta)$ \\
\hline S55D/E59D $\alpha \mathrm{PV}: \mathrm{Ca}^{2+}$ & & $-4.32 \pm 0.55$ & $-16.78 \pm 0.68$ \\
$\alpha \mathrm{PV}: \mathrm{Ca}^{2+}$ & $(-30.24 \pm 0.15)$ & $12.45 \pm 0.39$ & $0.00 \pm 0.56$ \\
D94S/G98E $\alpha \mathrm{PV}: \mathrm{Ca}^{2+}$ & & $40.84 \pm 0.37$ & $28.38 \pm 0.55$ \\
$\Delta \alpha \mathrm{PV}: \mathrm{Ca}^{2+}$ & $(-28.84 \pm 0.06)$ & $14.89 \pm 0.64$ & $2.43 \pm 0.75$ \\
& & $(1.40 \pm 0.16)$ & \\
S55D/E59D $\alpha \mathrm{PV}: \mathrm{Mg}^{2+}$ & & $14.60 \pm 0.59$ & $-28.74 \pm 0.72$ \\
$\alpha \mathrm{PV}: \mathrm{Mg}{ }^{2+}$ & $(0.64 \pm 0.17)$ & $43.34 \pm 0.40$ & $0.00 \pm 0.57$ \\
D94S/G98E $\alpha \mathrm{PV}: \mathrm{Mg}^{2+}$ & & $60.19 \pm 0.55$ & $16.85 \pm 0.68$ \\
$\Delta \alpha \mathrm{PV}: \mathrm{Mg}^{2+}$ & $(1.22 \pm 0.17)$ & $44.96 \pm 0.66$ & $1.62 \pm 0.78$
\end{tabular}

Data are included for the $\beta$-sheet residues (F57-E59, K96-G98). Error bars represent sem. Holo state energies are shown in parenthesis. 
Table S4: MM/GBSA reorganization energies of the D-helix residues.

\begin{tabular}{l|ccc}
\hline System & $\mathrm{G}_{R E}^{\text {Calc }}(\mathrm{D})$ & $\Delta \mathrm{G}_{R E}^{\text {Calc }}(\mathrm{D})$ & $\Delta \Delta \mathrm{G}_{R E}^{\text {Calc }}(\mathrm{D})$ \\
\hline $\mathrm{S} 55 \mathrm{D} / \mathrm{E} 59 \mathrm{D} \alpha \mathrm{PV}: \mathrm{Ca}^{2+}$ & & $23.79 \pm 0.29$ & $-3.76 \pm 0.35$ \\
$\alpha \mathrm{PV}: \mathrm{Ca}^{2+}$ & $(-149.00 \pm 0.17)$ & $27.55 \pm 0.18$ & $0.00 \pm 0.26$ \\
D94S/G98E $\alpha \mathrm{PV}: \mathrm{Ca}^{2+}$ & & $31.18 \pm 0.41$ & $3.62 \pm 0.45$ \\
$\Delta \alpha \mathrm{PV}: \mathrm{Ca}^{2+}$ & $(-137.67 \pm 0.14)$ & $34.45 \pm 1.83$ & $6.89 \pm 1.84$ \\
& & $(11.33 \pm 0.22)$ & \\
S55D/E59D $\alpha \mathrm{PV}: \mathrm{Mg}^{2+}$ & & $32.23 \pm 1.27$ & $-11.1 \pm 1.27$ \\
$\alpha \mathrm{PV}: \mathrm{Mg}^{2+}$ & $(-133.21 \pm 0.08)$ & $43.35 \pm 0.11$ & $0.00 \pm 0.16$ \\
D94S/G98E $\alpha \mathrm{PV}: \mathrm{Mg}^{2+}$ & & $42.65 \pm 0.36$ & $-0.71 \pm 0.38$ \\
$\Delta \alpha \mathrm{PV}: \mathrm{Mg}^{2+}$ & $(-126.82 \pm 0.06)$ & $45.29 \pm 1.82$ & $1.94 \pm 1.82$ \\
& & $(6.39 \pm 0.10)$ & \\
\hline
\end{tabular}

Residues E60 to F70 are considered for this calculation. All energies in kcal/mol. Holo state energies are shown in parenthesis. Error bars represent sem.

Table S5: MM/GBSA reorganization energies of the E-helix residues.

\begin{tabular}{l|ccc}
\hline System & $\mathrm{G}_{R E}^{\text {Calc }}(\mathrm{E})$ & $\Delta \mathrm{G}_{R E}^{\text {Calc }}(\mathrm{E})$ & $\Delta \Delta \mathrm{G}_{R E}^{C a l c}(\mathrm{E})$ \\
\hline $\mathrm{S} 55 \mathrm{D} / \mathrm{E} 59 \mathrm{D} \alpha \mathrm{PV}: \mathrm{Ca}^{2+}$ & & $-7.43 \pm 1.79$ & $-3.48 \pm 2.63$ \\
$\alpha \mathrm{PV}: \mathrm{Ca}^{2+}$ & $(-108.42 \pm 0.48)$ & $-3.95 \pm 1.92$ & $0.00 \pm 2.71$ \\
$\mathrm{D} 94 \mathrm{~S} / \mathrm{G} 98 \mathrm{E} \alpha \mathrm{PV}: \mathrm{Ca}^{2+}$ & & $-6.82 \pm 1.70$ & $-2.86 \pm 2.56$ \\
$\Delta \alpha \mathrm{PV}: \mathrm{Ca}^{2+}$ & $(-104.14 \pm 0.23)$ & $-8.50 \pm 0.61$ & $-4.55 \pm 2.01$ \\
& & $(4.28 \pm 0.53)$ & \\
S55D/E59D $\alpha \mathrm{PV}: \mathrm{Mg}^{2+}$ & & $-6.94 \pm 1.82$ & $0.25 \pm 2.64$ \\
$\alpha \mathrm{PV}: \mathrm{Mg}^{2+}$ & $(-111.67 \pm 0.42)$ & $-7.20 \pm 1.90$ & $0.00 \pm 2.69$ \\
D94S/G98E $\alpha \mathrm{PV}: \mathrm{Mg}^{2+}$ & & $-6.34 \pm 1.62$ & $0.86 \pm 2.50$ \\
$\Delta \alpha \mathrm{PV}: \mathrm{Mg}^{2+}$ & $(-104.79 \pm 0.22)$ & $-9.15 \pm 0.61$ & $-1.94 \pm 2.00$ \\
& & $(6.88 \pm 0.47)$ & \\
\hline
\end{tabular}

Residues E60 to F70 are considered for this calculation. All energies in kcal/mol. Holo state energies are shown in parenthesis. Error bars represent sem. 
Table S6: Estimate of effective oxygen charge $\left(\mathrm{q}_{e f f}\right)$ for each binding site configuration based on MD simulation.

\begin{tabular}{l|cccccc}
\hline Case & Loop & Backbone (-0.56e) & Hydroxyl (-0.65e) & Carbonyl (-0.81e) & Water $(-0.83 \mathrm{e})$ & qeff \\
\hline S55D/E59D & CD & 1.0 & 0.0 & 5.0 & 1.0 & $-0.77 \mathrm{e}$ \\
& $\mathrm{EF}$ & 1.0 & 0.0 & 5.0 & 1.0 & $-0.77 \mathrm{e}$ \\
$\alpha \mathrm{PV}$ & $\mathrm{CD}$ & 1.0 & 1.0 & 5.5 & 0.0 & $-0.75 \mathrm{e}$ \\
& $\mathrm{EF}$ & 1.0 & 0.0 & 5.5 & 1.0 & $-0.77 \mathrm{e}$ \\
$\mathrm{D} 94 \mathrm{~S} / \mathrm{G} 94 \mathrm{E}$ & $\mathrm{CD}$ & 1.0 & 1.0 & 5.0 & 0.0 & $-0.75 \mathrm{e}$ \\
& $\mathrm{EF}$ & 1.0 & 0.3 & 5.7 & 0.5 & $-0.76 \mathrm{e}$ \\
$\Delta \alpha \mathrm{PV}$ & $\mathrm{CD}$ & 1.0 & 1.0 & 5.0 & 0.0 & $-0.74 \mathrm{e}$ \\
& $\mathrm{EF}$ & 1.0 & 0.0 & 5.0 & 1.0 & $-0.77 \mathrm{e}$ \\
\hline
\end{tabular}

$\overline{q_{e f f}}$ is used for the MSA calculations. The partial charges of different oxygen types are adapted from the Amber ff14SB force field. Specifically, the backbone oxygen charge is from SER O atom type, the hydroxyl oxygen charge is from SER OG atom type, the carbonyl oxygen charge is from GLU OD atom type and water oxygen charge is from the TIP3P water O atom type.

Table S7: Contact frequency of ion and CD site residues.

\begin{tabular}{|c|c|c|c|c|c|c|}
\hline System & & & & & & \\
\hline$\alpha \mathrm{PV}: \mathrm{Ca}^{2+}$ & E62 $100 \%$ & E59 100\% & F57 100\% & D53 100\% & D51 $100 \%$ & S55 99\% \\
\hline$\alpha \mathrm{PV}: \mathrm{Mg}^{2+}$ & E62 100\% & E59 100\% & F57 100\% & D53 100\% & D51 100\% & S55 97\% \\
\hline $\mathrm{S} 55 \mathrm{D} / \mathrm{E} 59 \mathrm{D} \alpha \mathrm{PV}: \mathrm{Ca}^{2+}$ & E62 $100 \%$ & F57 100\% & D55 100\% & D53 100\% & D51 100\% & \\
\hline $\mathrm{S} 55 \mathrm{D} / \mathrm{E} 59 \mathrm{D} \alpha \mathrm{PV}: \mathrm{Mg}^{2+}$ & E62 100\% & F57 100\% & D55 100\% & D53 100\% & D51 100\% & \\
\hline D94S/G98E $\alpha \mathrm{PV}: \mathrm{Ca}^{2+}$ & E62 $100 \%$ & E59 100\% & F57 100\% & D53 100\% & D51 100\% & $\mathrm{S} 5599 \%$ \\
\hline D94S/G98E $\alpha \mathrm{PV}: \mathrm{Mg}^{2+}$ & E62 100\% & E59 100\% & D53 100\% & D51 $100 \%$ & F57 99\% & S55 98\% \\
\hline$\Delta \alpha \mathrm{PV}: \mathrm{Ca}^{2+}$ & E62 100\% & E59 100\% & F57 100\% & D53 100\% & D51 100\% & $\mathrm{S} 5599 \%$ \\
\hline$\Delta \alpha \mathrm{PV}: \mathrm{Mg}^{2+}$ & E62 100\% & E59 100\% & D53 100\% & D51 100\% & F57 99\% & $\mathrm{S} 5598 \%$ \\
\hline
\end{tabular}

Residues within $3 \AA$ of ions in the CD site are considered. Sorted from higher to the lower frequencies. Average of three sets of simulations are reported.

Table S8: Contact frequency of ion and EF site residues.

\begin{tabular}{|c|c|c|c|c|c|c|}
\hline System & & & & & & \\
\hline$\alpha \mathrm{PV}: \mathrm{Ca}^{2+}$ & K96 $100 \%$ & D94 100\% & D92 $100 \%$ & D90 $100 \%$ & E101 $100 \%$ & \\
\hline$\alpha \mathrm{PV}: \mathrm{Mg}^{2+}$ & K96 97\% & D94 100\% & D92 $100 \%$ & D90 $100 \%$ & E101 $100 \%$ & \\
\hline $\mathrm{S} 55 \mathrm{D} / \mathrm{E} 59 \mathrm{D} \alpha \mathrm{PV}: \mathrm{Ca}^{2+}$ & K96 100\% & D94 100\% & D92 100\% & D90 $100 \%$ & E101 $100 \%$ & \\
\hline $\mathrm{S} 55 \mathrm{D} / \mathrm{E} 59 \mathrm{D} \alpha \mathrm{PV}: \mathrm{Mg}^{2+}$ & K96 100\% & D94 100\% & D92 100\% & D90 100\% & E101 100\% & \\
\hline D94S/G98E $\alpha \mathrm{PV}: \mathrm{Ca}^{2+}$ & E98 $100 \%$ & D92 $100 \%$ & D90 100\% & E101 100\% & K96 99\% & S94 35\% \\
\hline D94S/G98E $\alpha \mathrm{PV}-\mathrm{Mg}^{2+}$ & E98 $100 \%$ & D92 $100 \%$ & D90 $100 \%$ & E101 100\% & K91 10\% & K96 1\% \\
\hline$\Delta \alpha \mathrm{PV}: \mathrm{Ca}^{2+}$ & K96 100\% & D94 100\% & D92 $100 \%$ & D90 100\% & E101 $100 \%$ & \\
\hline$\Delta \alpha \mathrm{PV}: \mathrm{Mg}^{2+}$ & D94 100\% & D92 100\% & D90 100\% & E101 100\% & K96 99\% & \\
\hline
\end{tabular}

Residues within $3 \AA$ of ions in the EF site are considered. Sorted from higher to the lower frequencies. Average of three sets of simulations are reported 


\section{S8.3 Supplemental Methods}

$$
\begin{aligned}
k_{B} T \ln \left(\rho_{i}(m+1)\right) & =k_{B} T \ln \left(\rho_{0, i}\right) \\
& +\mu_{0, i}^{e x}-z_{i} e_{0} \Psi(m)-\mu_{i e x}(m)
\end{aligned}
$$

In Eq. S1, $\rho_{i}$ is the density of the $\mathrm{i}$-th ion type in the binding site, $\rho_{0, i}$ is the density of the i-th ion in the bath (assumed to remain constant), $\mu_{i}^{e x}$ is the excess chemical potential of the i-th ion in the binding site, $\mu_{0, i}^{e x}$ is the excess chemical potential of the $\mathrm{i}$-th ion in the binding site (also constant), $z_{i}$ is the integer charge of the i-th ion species, $\Psi$ is the Donnan potential for the binding site, and $\mu_{i}^{e x}$ is the chemical potential of the i-th ion in the binding site.

\section{S8.4 Supplementary Results}

Protein backbone RMSDs To assess the variability of other simulated systems with the experimental crystal structures and also to compare and contrast between different systems studied, we calculated the backbone RMSDs of protein. All the ion bound forms converged around $1 \AA(\approx 2 \AA$ for $\Delta \alpha \mathrm{PV})$ in all the ion bound forms approximately after $100 \mathrm{~ns}$, where as the apo forms around $2 \AA$ after $600 \mathrm{~ns}(\approx 5 \AA$ for $\Delta \alpha \mathrm{PV})$. The backbone RMSDs shows that the presence of ions $\left(\mathrm{Ca}^{2+}\right.$ or $\left.\mathrm{Mg}^{2+}\right)$ dampen the structural fluctuations of the variants compared to the apo forms (Fig. S1). While the difference between the apo and ion bound forms is statistically significant in all PV variants studied, no statistical difference between $\mathrm{Ca}^{2+}$ and $\mathrm{Mg}^{2+}$ bound forms was evident. Additionally, RMSD calculations suggest that the AB domain stabilizes the intact complex as its holo state RMSD is considerably smaller than the $\Delta \alpha \mathrm{PV}(\approx 5 \AA$ for truncated apo vs. the $\approx 2 \AA$ for the other apo systems and $\approx 2 \AA$ for truncated ion bound vs. $\approx 1 \AA$ for other ion bound forms). Other pronounced structural variations are discussed further in sections 3.3. Overall, the structural similarities and converged RMSDs suggest that our simulation protocol can reproduce the conformational ensembles of ion-bound forms and also likely accurately model the apo-forms of the $\alpha \mathrm{PV}$ variants for which no structural data are available.

Water coordination of $\mathrm{Ca}^{2+}$ We additionally assessed the number of waters coordinated by $\mathrm{Ca}^{2+}$ while bound to the $\mathrm{CD}$ and EF hand loops (Fig. 2E-H). We relate the cumulative number of protein and water oxygens in panels Fig. 2I-J to determine the total coordination number of $\mathrm{Ca}^{2+}$. In the CD hand, only the $\mathrm{S} 55 \mathrm{D} / \mathrm{E} 59 \mathrm{D} \alpha \mathrm{PV}$ variant (Fig. 2G) reflects a bound water, which is roughly $2.2 \AA$ from the $\mathrm{Ca}^{2+}$. In this capacity, the bound water in S55D/E59D $\alpha \mathrm{PV}$ may help offset the loss of interaction of a bidentate residue E59 and coordinate D59 with the ion. In the EF site, all $\alpha \mathrm{PV}$ structures except D94S/G98E $\alpha \mathrm{PV}$ present one bound water in the coordination site Fig. 2H. The D94S/G98E $\alpha \mathrm{PV}$ count is 0.5, signifying that a single water is bound for about one-half of the simulation time.

Coordination shell in $\mathrm{Mg}^{2+}$ bound systems In the $\mathrm{Mg}^{2+}$ bound systems we demonstrate through radial distribution of protein oxygens that the proteins bind tighter to the $\mathrm{Mg}^{2+}$ ion compared to the Ca ${ }^{2+}$. In 
either binding site, the first oxygen coordination shell is at $\approx 1.7 \AA$ for all the four systems $(0.5 \AA$ less than the $\mathrm{Ca}^{2+}$ bound systems) (Fig. $2 \mathrm{E}, \mathrm{F}$ ). This indicates that protein has to rearrange to interact with the ions (compared to $\mathrm{Ca}^{2+}$ ), which might come at a thermodynamics cost. The number of coordinating oxygens in this CD site for the S55D/E59D $\alpha \mathrm{PV}$ is five, where as for all other systems it is six (Fig. 2G). In the EF site, five oxygens are coordinating with $\mathrm{Mg}^{2+}$ in all four systems (Fig. $2 \mathrm{H}$ ). Overall, we show that, on average, two fewer oxygens are coordinating the $\mathrm{Mg}^{2+}$ (in both sites combined) compared to $\mathrm{Ca}^{2+}$ bound systems, which partly explains the lower affinity of $\mathrm{Mg}^{2+}$ to $\alpha \mathrm{PV}$ systems compared to $\mathrm{Ca}^{2+}$. This is in line with the other earlier observations that the $\mathrm{Mg}^{2+}$ coordination is lower than $\mathrm{Ca}^{2+}$ in $\mathrm{PVs}$ [37]. Moreover, the water coordination in the $\mathrm{Mg}^{2+}$ bound systems is very similar to the $\mathrm{Ca}^{2+}$ bound ones, except that the first hydration shell is closer in the $\mathrm{Mg}^{2+}$ bound systems compared to the $\mathrm{Ca}^{2+}$ bound systems $(\approx 0.5 \AA$ lower $)$ (Fig. 2A-D).

For $\mathrm{S} 55 \mathrm{D} / \mathrm{E} 59 \mathrm{D} \alpha \mathrm{PV}$, the $\Delta \Delta \mathrm{G}_{R E}^{C a l c}(\mathrm{CD})$ values attributed to the $\mathrm{CD}$ loop residues (L50-E62) summed to $\approx-8 \mathrm{kcal} / \mathrm{mol}$ (see Table S2), suggesting that $\mathrm{Ca}^{2+}$ binding is more favorable in the S55D/E59D $\alpha \mathrm{PV}$ site relative to the WT. This is not unexpected, given that the S55D substitution introduces an additional unit negative charge into the binding site. Meanwhile, $\Delta \Delta \mathrm{G}_{R E}^{C a l c}(\mathrm{CD})$ for D94S $/ \mathrm{G} 98 \mathrm{E} \alpha \mathrm{PV}$ is $\approx 13 \mathrm{kcal} / \mathrm{mol}$, which we found surprising given that the amino acid substitutions reside in the adjacent EF hand. Lastly, the score difference between the $\Delta \alpha \mathrm{PV}$ and WT holo states was approximately $\approx 12 \mathrm{kcal} / \mathrm{mol}$, which suggests $\mathrm{Ca}^{2+}$ binding was less favorable for the truncated species. Similarly, $\Delta \Delta \mathrm{G}_{R E}^{C \text { Calc }}(\mathrm{EF})$ for the EF hand residues spanning D90 to F102 were $\approx 14 \mathrm{kcal} / \mathrm{mol}$ for the S55D/E59D $\alpha \mathrm{PV}$ suggesting that the $\mathrm{Ca}^{2+}$ binding in this site is unfavorable; where as for the D94S/G98E $\alpha \mathrm{PVit}$ is $\approx 38 \mathrm{kcal} / \mathrm{mol}$ suggesting the unfavorable binding of $\mathrm{Ca}^{2+}$, which trends correctly with the experimental data. Altogether, the $\Delta \Delta \mathrm{G}_{R E}^{C a l c}$ contributions arising from both $\mathrm{CD}$ and $\mathrm{EF}$ hands were $\approx 6 \mathrm{kcal} / \mathrm{mol}$ for S55D $/ \mathrm{E} 59 \mathrm{D} \alpha \mathrm{PV}$ and $\approx 51 \mathrm{kcal} / \mathrm{mol}$ for D94S $/ \mathrm{G} 98 \mathrm{E}$ $\alpha \mathrm{PV}$. Nevertheless, these scores from the CD and EF hands did not consistently rank the variants by affinity Table S2, which suggests that non-local contributions play a significant role in the binding scores.

Specific hydrogen bonding interactions The solvation differences between apo and holo states are driven by conformational changes upon ion binding. We therefore analyzed hydrogen bonds between amino acid side chains to determine if any may help stabilize the holo state. In Fig. S9 we report that for the hydrogen bonds that change between the apo and holo states, a greater number are formed during $\mathrm{Ca}^{2+}$ binding as opposed to breaking. This includes the formation of S55-E59 (CD site) and K96-D94 (EF site) hydrogen bonds in the holo forms and two hydrogen bonds that are lost upon ion binding in all systems; (S65-D76 that connects D-helix with the loop that is bridging D and E helices and S55-D51 that form part of the CD loop) (Fig. S9). However, in the S55D/E59D $\alpha \mathrm{PV}$ interactions of S55 with E59 and D51 do not exist since the residues S55 and E59 were mutated to D55 and D59, respectively (Fig. S9). The D94S mutation in D94S/G98E $\alpha$ PV abolished the K96-D94 interaction, which we speculate results in high mobility of the EF site loop. Instead, a hydrogen bond between S94 and D90 is formed. In the $\Delta \alpha \mathrm{PV}$ system, the lack of $\mathrm{AB}$ negates the S78-D22 interaction. 
Glycine and glutamic acid play significant roles in tuning the dynamics of $\alpha P V$ helicity In this study we observed that amino acid type and the location are very critical in $\alpha \mathrm{PV}$. Proline and glycine are typical helix breakers $[68,50]$. Our data show glycine significantly increase dynamics of $\alpha \mathrm{PV}$ proteins as 4 out of 10 residues in the EF domain loop are glycine. For this reason the EF domain loop is highly flexible (glycines are the most flexible of all residues[50]) compared to the CD site loop in all $\alpha \mathrm{PVs}$, including the $\mathrm{S} 55 \mathrm{D} / \mathrm{E} 59 \mathrm{D} \alpha \mathrm{PV}$. The instability of the EF site loop due to the presence of extra glycine and aspartic acids is also reflected in the MM/GBSA reorganization energies As shown in Table $\mathrm{S} 2$, the $\Delta \mathrm{G}_{R E}^{C a l c}$ for the $\mathrm{CD}$ site loop residues is consistently more favorable than the EF site residues in all variants studied by at least 50 $\mathrm{kcal} / \mathrm{mol}$. Additionally, there are two glycines in the D-helix at positions 64 and 69. In particular, G64 is acting as a helix breaker in the D-helix as the helicity of residues from $65-70$ is reduced by $\approx 35 \%$ (Fig. $5 \mathrm{C}$ ). The D-helix is part of the $\alpha \mathrm{PV}$ hydrophobic core and interacts directly with the cation, therefore, cation binding in the CD site counter the effects of G64 and imparts stability to D-helix. Hence it is apparent that glycine increases the dynamics of $\alpha \mathrm{PV}$ helicity and therefore may be a mechanism for increasing affinity. 


\section{S8.5 Limitations}

Limitations of our approach include the limited MD simulation length (microsecond), in addition to approximations associated with the MD and MM/GBSA methods (detailed in the review [49]). The time scale of binding, folding and unfolding etc. are usually on the order of milliseconds to seconds [85], compared to our microsecond-length simulations. The other significant limitation is that MM/GBSA is not sufficiently accurate to predict the solvation energies of ions, which is crucial to assess the absolute/relative binding affinities of the ions to $\alpha \mathrm{PV}$ [49]. We attribute this to the inherent approximations in this end point method, such as the implicit treatment of solvent, neglect of entropy, and the sensitivity of the method to the solute dielectric constant and the challenges in accurately modeling the non-polar contribution to the solution free energy $[86,87,88]$. Force field deficiencies might also contribute to the inaccuracies in the binding affinity estimation, particularly those pertaining to describing interactions with divalent ions like $\mathrm{Ca}^{2+}[89,29]$.

Although, we used $\Delta \Delta \mathrm{G}_{R E}^{C a l c}$ values from the MM/GBSA analysis to rank order all the variants according to their affinities, we omitted the apo state of $\Delta \alpha \mathrm{PV}$ for these comparisons. We believe $\Delta \alpha \mathrm{PV}$ was problematic because this simulation was started from the completely ordered holo structure by removing $\mathrm{Ca}^{2+}$, since the apo state crystal structure of the $\Delta \alpha \mathrm{PV}$ was unavailable; experimental studies report that the $\Delta \alpha \mathrm{PV}$ apo structure is completely disordered [18]. However, in our simulations we only observed partial unfolding of the structure, which warrants more simulation time that is beyond the scope of this study.

We also reported in Section 3.1 correlations between RMSF of the EF site loop residues and the affinity of ions for $\mathrm{Ca}^{2+}$. Although we could provide estimates for the enthalpy of the conformations comprising the apo and holo ensembles, the entropic contributions were inconclusive. We anticipate there are two significant contributions to entropy changes, including the apparent decrease in configurational entropy of the holo state relative to the apo state suggested by the RMSFs and the anticipated increase in solvent configuration entropy from desolvation of EF hand. It has been suggested that the increase in solvent configurational entropy likely predominates the loss of entropy from decreased conformational fluctuations [47].

Lastly, our MSA model indicates that the chemical potential is rather insensitive to the number of bound oxygens ( 8 is favored over 5 by less than $1 \mathrm{kcal} / \mathrm{mol}$ ). It is important to recognize that our MSA implementation does not account for quantum effects like polarization or orbital geometries that could favor certain coordination numbers and geometries [38, 54]. 


\section{Bibliography}

[1] B Schwaller. The continuing disappearance of "pure" Ca2+ buffers. Cellular and Molecular Life Sciences, $66(2): 275-300,2009.2$

[2] Michael T Henzl and Sayeh Agah. Divalent ion-binding properties of the two avian beta-parvalbumins. Proteins-Structure Function And Bioinformatics, 62(1):270-278, 2005. 2

[3] Michael T Henzl, Meredith E Davis, and Anmin Tan. Leucine 85 Is an Important Determinant of Divalent Ion Affinity in Rat $\beta$-Parvalbumin (Oncomodulin) . Biochemistry, 47(51):13635-13646, 2008. 2

[4] Michael T Henzl, Sayeh Agah, and John D Larson. Association of the AB and CD-EF Domains from Rat $\alpha$ - and $\beta$-Parvalbumin . Biochemistry, 43(34):10906-10917, 2004. 2

[5] Sayeh Agah, John D Larson, and Michael T Henzl. Impact of Proline Residues on Parvalbumin Stability . Biochemistry, 42(37):10886-10895, 2003. 2

[6] Joseph Markowitz, Richard R Rustandi, Kristen M Varney, Paul T Wilder, Ryan Udan, Su Ling Wu, William DeW Horrocks, and David J Weber. Calcium-binding properties of wild-type and EF-hand mutants of $\mathrm{S} 100 \mathrm{~B}$ in the presence and absence of a peptide derived from the C-terminal negative regulatory domain of p53. Biochemistry, 44(19):7305-7314, 2005. 2

[7] Melissa A Liriano, Kristen M Varney, Nathan T Wright, Cassandra L Hoffman, Eric A Toth, Rieko Ishima, and David J Weber. Target binding to S100B reduces dynamic properties and increases Ca $(2+)$-binding affinity for wild type and EF-hand mutant proteins. Journal Of Molecular Biology, 423(3):365-385, 2012. 2

[8] Chee Chew Lim, Haijun Yang, Mingfeng Yang, Chien-Kao Wang, Jianru Shi, Eric A Berg, David R Pimentel, Judith K Gwathmey, Roger J Hajjar, Michiel Helmes, Catherine E Costello, Shuanghong Huo, and Ronglih Liao. A novel mutant cardiac troponin $\mathrm{C}$ disrupts molecular motions critical for calcium binding affinity and cardiomyocyte contractility. Biophysical Journal, 94(9):3577-3589, 2008. 2

[9] Michelle S Parvatiyar, Jose Renato Pinto, Jingsheng Liang, and James D Potter. Predicting cardiomyopathic phenotypes by altering the Ca2+ affinity of cardiac troponin C. The Journal of biological chemistry, 285(36):27785-27797, 2010. 2

[10] S B Tikunova, J A Rall, and J P Davis. Effect of hydrophobic residue substitutions with glutamine on Ca2+ binding and exchange with the N-domain of troponin C. Biochemistry, 41(21):6697-6705, 2002.2

[11] Jianchao Zhang, Vikram Shettigar, George C Zhang, Daniel G Kindell, Xiaotong Liu, Joseph J Lopez, Vinatham Yerrimuni, Grace A Davis, and Jonathan P Davis. Engineering Parvalbumin for the Heart: Optimizing the Mg Binding Properties of Rat $\beta$-Parvalbumin. Frontiers in Physiology, 2:77, 2011. 2 
[12] Wang Wang, Matthew S Barnabei, Michelle L Asp, Frazer I Heinis, Erik Arden, Jennifer Davis, Elizabeth Braunlin, Qi Li, Jonathan P Davis, and James D Potter. Noncanonical EF-hand motif strategically delays Ca2+ buffering to enhance cardiac performance. Nature medicine, 19(3):305-312, 2013. 2

[13] David W Rodenbaugh, Wang Wang, Jennifer Davis, Terri Edwards, James D Potter, and Joseph M Metzger. Parvalbumin isoforms differentially accelerate cardiac myocyte relaxation kinetics in an animal model of diastolic dysfunction. American Journal of Physiology-Heart and Circulatory Physiology, 293 (3):H1705-H1713, 2007. 2

[14] Michael T Henzl, John D Larson, and Sayeh Agah. Influence of monovalent cation identity on parvalbumin divalent ion-binding properties. Biochemistry, 43(10):2747-2763, 2004. 2, 1

[15] Yong-Hwan Lee, John J. Tanner, John D. Larson, and Michael T. Henzl. Crystal Structure of a High-Affinity Variant of Rat $\alpha$-Parvalbumin $<\sup ></ \sup >$. Biochemistry, 43(31):10008-10017, August 2004. ISSN 0006-2960. doi: 10.1021/bi0492915. 2, 3, 1, 6.1, S1

[16] John J Tanner, Sayeh Agah, Yong-Hwan Lee, and Michael T Henzl. Crystal Structure of the D94S/G98E Variant of Rat $\alpha$-Parvalbumin. An Explanation for the Reduced Divalent Ion Affinity . Biochemistry, 44 (33):10966-10976, 2005. 2, 3, 1, 6.1, S1

[17] K M Elkins and P Z Gatzeva-Topalova. Molecular dynamics study of Ca2+ binding loop variants of parvalbumin with modifications at thegateway'position. Protein engineering, 2001. 2, 4.3

[18] Michel Thépaut, Marie Paule Strub, Adrien Cavé, Jean Louis Banères, Martin W Berchtold, Christian Dumas, and André Padilla. Structure of rat parvalbumin with deleted AB domain: Implications for the evolution of EF hand calciumbinding proteins and possible physiological relevance. Proteins-Structure Function And Bioinformatics, 45(2):117-128, 2001. 2, 3, 3.2, 1, 4.3, 6.1, S4, S1, S8.5

[19] H H Kawasaki, S S Nakayama, and R H R H Kretsinger. Classification and evolution of EF-hand proteins. BioMetals, 11(4):277-295, 1998. 2

[20] Jessica L Gifford, Michael P Walsh, and Hans J Vogel. Structures and metal-ion-binding properties of the Ca2+-binding helix-loop-helix EF-hand motifs. The Biochemical journal, 405(2):199-221, 2007. 2, $3.4,4.3$

[21] Zenon Grabarek. Structural Basis for Diversity of the EF-hand Calcium-binding Proteins. Journal Of Molecular Biology, 359(3):509-525, 2006. 2, 3.4, 4.2, 4.3

[22] Zenon Grabarek. Insights into modulation of calcium signaling by magnesium in calmodulin, troponin C and related EF-hand proteins. BBA - Molecular Cell Research, 1813(5):913-921, 2011. 2, 4.2, 4.3, 4.4 
[23] Yubin Zhou, Wei Yang, Michael Kirberger, Hsiau-Wei Lee, Gayatri Ayalasomayajula, and Jenny J Yang. Prediction of EF-hand calcium-binding proteins and analysis of bacterial EF-hand proteins. Proteins-Structure Function And Bioinformatics, 65(3):643-655, 2006. 2

[24] Xue Wang, Michael Kirberger, Fasheng Qiu, Guantao Chen, and Jenny J Yang. Towards predicting Ca 2+binding sites with different coordination numbers in proteins with atomic resolution. Proteins-Structure Function And Bioinformatics, 75(4):787-798, 2009. 2

[25] Michael Kirberger, Xue Wang, Hai Deng, Wei Yang, Guantao Chen, and Jenny J Yang. Statistical analysis of structural characteristics of protein Ca2+-binding sites. JBIC Journal of Biological Inorganic Chemistry, 13(7):1169-1181, 2008. 2

[26] Zhifeng Jing, Chengwen Liu, Rui Qi, and Pengyu Ren. Many-body effect determines the selectivity for ca2+ and mg2+ in proteins. Proceedings of the National Academy of Sciences, 115(32):E7495-E7501, 2018. $2,4.1,4.4$

[27] Kalyan Immadisetty, Jeevapani Hettige, and Mahmoud Moradi. What can and cannot be learned from molecular dynamics simulations of bacterial proton-coupled oligopeptide transporter gkpot? The Journal of Physical Chemistry B, 121(15):3644-3656, 2016. 2

[28] S Marchand and B Roux. Molecular dynamics study of calbindin D9k in the apo and singly and doubly calcium-loaded states. Proteins-Structure Function And Bioinformatics, 33(2):265-284, 1998. 2

[29] Pengfei Li, Benjamin P Roberts, Dhruva K Chakravorty, and Kenneth M Merz. Rational Design of Particle Mesh Ewald Compatible Lennard-Jones Parameters for +2 Metal Cations in Explicit Solvent. Journal of Chemical Theory and Computation, 9(6):2733-2748, 2013. 2, 6.2, S8.5

[30] Akansha Saxena and David Sept. Multisite Ion Models That Improve Coordination and Free Energy Calculations in Molecular Dynamics Simulations. Journal of Chemical Theory and Computation, 9(8): 3538-3542, 2013. 2

[31] P.M. Kekenes-Huskey, V.T. Metzger, B.J. Grant, and J.A. McCammon. Calcium binding and allosteric signaling mechanisms for the sarcoplasmic reticulum Ca2+ ATPase. Protein Science, 21(10):1429-1443, 2012. ISSN 09618368 1469896X. doi: 10.1002/pro.2129. 2

[32] P M Kekenes-Huskey, A. Gillette, J. Hake, and J Andrew McCammon. Finite Element Estimation of Protein-Ligand Association Rates with Post-Encounter Effects: Applications to Calcium binding in Troponin C and SERCA. Computational science \& discovery, 5(1):0-20, 2012. ISSN 1749-4699. doi: 10.1088/1749-4699/5/1/014015. 2

[33] Wei Jiang, Yun Luo, Luca Maragliano, and Benoît Roux. Calculation of Free Energy Landscape in Multi-Dimensions with Hamiltonian-Exchange Umbrella Sampling on Petascale Supercomputer. J CHEM THEORY COMPUT, 8(11):4672-4680, 2012. 2 
[34] L Michel Espinoza-Fonseca, Joseph M Autry, and David D Thomas. Microsecond molecular dynamics simulations of Mg- and K-bound E1 intermediate states of the calcium pump. PLoS ONE, 9(4): e95979-e95979, 2014. 2

[35] Y. Cheng, S. Lindert, P. Kekenes-Huskey, V.S. Rao, R.J. Solaro, P.R. Rosevear, R. Amaro, A.D. McCulloch, J.A. McCammon, and M. Regnier. Computational studies of the effect of the S23D/S24D troponin i mutation on cardiac troponin structural dynamics. Biophysical Journal, 107(7):1675-1685, 2014. ISSN 15420086 00063495. doi: 10.1016/j.bpj.2014.08.008. 2

[36] S. Lindert, Y. Cheng, P. Kekenes-Huskey, M. Regnier, and J.A. McCammon. Effects of HCM cTnI mutation R145G on troponin structure and modulation by PKA phosphorylation elucidated by molecular dynamics simulations. Biophysical Journal, 108(2):395-407, 2015. ISSN 1542008600063495 . doi: 10.1016/j.bpj.2014.11.3461. 2, 3.1

[37] M Susan Cates, Miguel L Teodoro, and George N Phillips. Molecular Mechanisms of Calcium and Magnesium Binding to Parvalbumin. Biophysical Journal, 82(3):1133-1146, 2002. 2, 4.3, 4.4, S8.4

[38] Amir N Kucharski, Caitlin E Scott, Jonathan P. Davis, and Peter M Kekenes-Huskey. Understanding Ion Binding Affinity and Selectivity in $\beta$-Parvalbumin Using Molecular Dynamics and Mean Spherical Approximation Theory. The Journal of Physical Chemistry B, 120(33):8617-8630, August 2016. ISSN 1520-6106. doi: 10.1021/acs.jpcb.6b02666. 2, 3.1, 3, 4.2, 6.4, S8.5

[39] Yun Zhao, Jianfeng He, and Jing Li. The structural modeling of ef-hand motifs in parvalbumin. Journal of Mathematical Chemistry, 56(8):2525-2536, 2018. 2

[40] Christopher A Bottoms, Jonathan P Schuermann, Sayeh Agah, Michael T Henzl, and John J Tanner. Crystal structure of rat $\alpha$-parvalbumin at 1.05 å resolution. Protein science, 13(7):1724-1734, 2004. 3, $3.1,6.1$

[41] Michael T Henzl and John J Tanner. Solution structure of Ca2+free rat $\alpha$ parvalbumin. Protein Science, 17(3):431-438, 2008. 3.1

[42] S. Lindert, P.M. Kekenes-Huskey, and J.A. McCammon. Long-timescale molecular dynamics simulations elucidate the dynamics and kinetics of exposure of the hydrophobic patch in troponin C. Biophysical Journal, 103(8):1784-1789, 2012. ISSN 00063495 15420086. doi: 10.1016/j.bpj.2012.08.058. 3.1

[43] Walter Rocchia, Emil Alexov, and Barry Honig. Extending the applicability of the nonlinear poissonboltzmann equation: multiple dielectric constants and multivalent ions. The Journal of Physical Chemistry B, 105(28):6507-6514, 2001. 3.2

[44] Shenggao Zhou, Zhongming Wang, and Bo Li. Mean-field description of ionic size effects with nonuniform ionic sizes: A numerical approach. Physical Review E, 84(2):021901, August 2011. ISSN 1539-3755. doi: 10.1103/PhysRevE.84.021901. 3.2 
[45] Michael T Henzl, Sayeh Agah, and John D Larson. Characterization of the Metal Ion-Binding Domains from Rat $\alpha$ - and $\beta$-Parvalbumins . Biochemistry, 42(12):3594-3607, 2003. 1, S1

[46] Wolfgang Nonner, Dirk Gillespie, Douglas Henderson, and Bob Eisenberg. Ion Accumulation in a Biological Calcium Channel: Effects of Solvent and Confining Pressure. The Journal of Physical Chemistry B, 105(27):6427-6436, 2001. ISSN 10895647. doi: 10.1021/jp010562k. 3.3, 6.4

[47] Jessica L Gifford, Michael P Walsh, and Hans J Vogel. Structures and metal-ion-binding properties of the ca2+-binding helix-loop-helix ef-hand motifs. Biochemical Journal, 405(2):199-221, 2007. 3.4, S8.5

[48] David Allouche, Joseph Parello, and Yves-Henri Sanejouand. Ca2+/mg2+ exchange in parvalbumin and other ef-hand proteins. a theoretical study. Journal of molecular biology, 285(2):857-873, 1999. 4.1

[49] Kalyan Immadisetty and Jeffry D Madura. A review of monoamine transporter-ligand interactions. Current computer-aided drug design, 9(4):556-568, 2013. 4.1, S8.5

[50] Anatoly M Ruvinsky and Ilya A Vakser. Sequence composition and environment effects on residue fluctuations in protein structures. The Journal of chemical physics, 133(15):10B609, 2010. 4.2, 4.3, S8.4

[51] Wendy S VanScyoc and Madeline A Shea. Phenylalanine fluorescence studies of calcium binding to n-domain fragments of paramecium calmodulin mutants show increased calcium affinity correlates with increased disorder. Protein Science, 10(9):1758-1768, 2001. 4.2

[52] Alisa A Vologzhannikova, Polina A Khorn, Alexei S Kazakov, Ramis G Ismailov, Andrei S Sokolov, Vladimir N Uversky, Eugene A Permyakov, and Sergei E Permyakov. In search for globally disordered apo-parvalbumins: Case of parvalbumin $\beta$-1 from coho salmon. Cell calcium, 67:53-64, 2017.4 .2

[53] Ric Michael Procyshyn and Ronald E Reid. A structure/activity study of calcium affinity and selectivity using a synthetic peptide model of the helix-loop-helix calcium-binding motif. Journal of Biological Chemistry, 269(3):1641-1647, 1994. 4.2

[54] Bin Sun, Bradley D Stewart, Amir N Kucharski, and Peter M Kekenes-Huskey. Thermodynamics of cation binding to the sarcoendoplasmic reticulum calcium atpase pump and impacts on enzyme function. Journal of chemical theory and computation, 15(4):2692-2705, 2019. 4.2, S8.5

[55] David C LaPorte, Berta M Wierman, and Daniel R Storm. Calcium-induced exposure of a hydrophobic surface on calmodulin. Biochemistry, 19(16):3814-3819, 1980. 4.3

[56] Brenda R Sorensen, Laurel A Faga, Rainbo Hultman, and Madeline A Shea. An interdomain linker increases the thermostability and decreases the calcium affinity of the calmodulin n-domain. Biochemistry, 41(1):15-20, 2002. 4.3 
[57] P.M. Kekenes-Huskey, S. Lindert, and J.A. McCammon. Molecular Basis of Calcium-Sensitizing and Desensitizing Mutations of the Human Cardiac Troponin C Regulatory Domain: A Multi-Scale Simulation Study. PLoS Computational Biology, 8(11), 2012. ISSN 1553734X 15537358. doi: 10.1371/journal.pcbi. 1002777. 4.3

[58] Marta Oleszczuk, Ian M Robertson, Monica X Li, and Brian D Sykes. Solution structure of the regulatory domain of human cardiac troponin $\mathrm{C}$ in complex with the switch region of cardiac troponin I and W7: The basis of W7 as an inhibitor of cardiac muscle contraction. Journal of molecular and cellular cardiology, 48(5):925-933, 2010. 4.3

[59] Nathan T Wright, Brian R Cannon, Paul T Wilder, Michael T Morgan, Kristen M Varney, Danna B Zimmer, and David J Weber. Solution Structure of S100A1 Bound to the CapZ Peptide (TRTK12). Journal Of Molecular Biology, 386(5):1265-1277, 2009. 4.3

[60] Caitlin E Scott and Peter M Kekenes-Huskey. Molecular Basis of S100A1 Activation at Saturating and Subsaturating Calcium Concentrations. Biophysical Journal, 110(5):1052-1063, 2016. 4.3

[61] Bin Sun and Peter M. Kekenes-Huskey. Molecular Basis of S100A1 Activation and Target Regulation Within Physiological Cytosolic Ca2+ Levels. Frontiers in Molecular Biosciences, 7:77, jun 2020. ISSN 2296889X. doi: 10.3389/fmolb.2020.00077. URL https://www.ncbi.nlm.nih.gov/pmc/articles/ PMC7324869/. 4.3

[62] O B Peersen, T S Madsen, and J J Falke. Intermolecular tuning of calmodulin by target peptides and proteins: differential effects on $\mathrm{Ca} 2+$ binding and implications for kinase activation. Protein Science, 6 (4):794-807, 1997. 4.3

[63] A Carl Whittington and Timothy S Moerland. Resurrecting prehistoric parvalbumins to explore the evolution of thermal compensation in extant antarctic fish parvalbumins. Journal of Experimental Biology, 215(18):3281-3292, 2012. 4.3

[64] Natalie CJ Strynadka and Michael NG James. Crystal structures of the helix-loop-helix calcium-binding proteins. Annual review of biochemistry, 58(1):951-999, 1989. 4.3

[65] Gary S Shaw, Robert S Hodges, and Brian D Sykes. Calcium-induced peptide association to form an intact protein domain: $1 \mathrm{~h} \mathrm{nmr} \mathrm{structural} \mathrm{evidence.} \mathrm{Science,} \mathrm{249(4966):280-283,} \mathrm{1990.} 4.3$

[66] Melanie R Nelson and Walter J Chazin. Structures of ef-hand ca 2+-binding proteins: Diversity in the organization, packing and response to ca 2+ binding. Biometals, 11(4):297-318, 1998. 4.3

[67] Monique Laberge, Wayne W Wright, Katakam Sudhakar, Paul A Liebman, and Jane M Vanderkooi. Conformational effects of calcium release from parvalbumin: comparison of computational simulations with spectroscopic investigations. Biochemistry, 36(18):5363-5371, 1997. 4.3 
[68] Kenichiro Imai and Shigeki Mitaku. Mechanisms of secondary structure breakers in soluble proteins. Biophysics, 1:55-65, 2005. 4.3, S8.4

[69] Martin Lepšík and Martin J Field. Binding of Calcium and Other Metal Ions to the EF-Hand Loops of Calmodulin Studied by Quantum Chemical Calculations and Molecular Dynamics Simulations. The Journal of Physical Chemistry B, 111(33):10012-10022, 2007. 4.4

[70] L Blum. Solution of the mean spherical approximation for hard ions and dipoles of arbitrary size. Journal of Statistical Physics, 18(5):451-474, 1978. 4.4

[71] JOHN R Dedman, JAMES D Potter, RICHARD L Jackson, J DAVID Johnson, and ANTHONY R Means. Physicochemical properties of rat testis ca2+-dependent regulator protein of cyclic nucleotide phosphodiesterase. relationship of ca2+-binding, conformational changes, and phosphodiesterase activity. Journal of Biological Chemistry, 252(23):8415-8422, 1977. 4.4

[72] Wang Wang, Matthew S Barnabei, Michelle L Asp, Frazer I Heinis, Erik Arden, Jennifer Davis, Elizabeth Braunlin, Qi Li, Jonathan P Davis, James D Potter, et al. Noncanonical ef-hand motif strategically delays ca $2+$ buffering to enhance cardiac performance. Nature medicine, 19(3):305-312, 2013. 5

[73] David Case, Robin Betz, D.S. Cerutti, Thomas Cheatham, Thomas Darden, Robert Duke, T.J. Giese, Holger Gohlke, Andreas Gtz, Nadine Homeyer, Saeed Izadi, Pawel Janowski, J Kaus, Andriy Kovalenko, Tai-Sung Lee, S LeGrand, P Li, C Lin, Tyler Luchko, and Peter A. Kollman. Amber 16. University of California: San Francisco, CA, 04 2016. doi: 10.13140/RG.2.2.27958.70729. 6.1, 6.2

[74] William L Jorgensen, Jayaraman Chandrasekhar, Jeffry D Madura, Roger W Impey, and Michael L Klein. Comparison of simple potential functions for simulating liquid water. The Journal of Chemical Physics, 79(2):926-935, 1983. doi: 10.1063/1.445869. URL https://doi.org/10.1063/1.445869. 6.2

[75] David Case, Thomas Darden, Thomas E. Cheatham, Carlos Simmerling, Junmei Wang, Robert E. Duke, Ray Luo, Ross C. Walker, Wei Zhang, and Kenneth M. Merz. Amber 12. University of California: San Francisco, $C A, 2012$. 6.2

[76] H. J.C. Berendsen, J. P.M. Postma, W. F. Van Gunsteren, A. Dinola, and J. R. Haak. Molecular dynamics with coupling to an external bath. The Journal of Chemical Physics, 81(8):3684-3690, 1984. ISSN 00219606. doi: 10.1063/1.448118. URL https://doi.org/10.1063/1.448118. 6.2

[77] Tom Darden, Darrin York, and Lee Pedersen. Particle mesh Ewald: An Nlog(N) method for Ewald sums in large systems. The Journal of chemical physics, 98(12):10089-10092, 1993. 6.2

[78] Jean-Paul Ryckaert, Giovanni Ciccotti, and Herman J C Berendsen. Numerical integration of the cartesian equations of motion of a system with constraints: molecular dynamics of $<\mathrm{i}>\mathrm{n}</ \mathrm{i}>$-alkanes. Journal of Computational Physics, 23(3):327-341, 1977. 6.2 
[79] W Humphrey, A Dalke, and K Schulten. VMD: visual molecular dynamics. Journal of molecular graphics, $14(1): 33-38,1996.6 .3$

[80] Masoumeh Yahyavi, Sajad Falsafi-Zadeh, Zahra Karimi, Giti Kalatarian, and Hamid Galehdari. Vmdss: A graphical user interface plug-in to calculate the protein secondary structure in vmd program. Bioinformation, 10(8):548, 2014. 6.3

[81] Daniel R. Roe and Thomas E. Cheatham. PTRAJ and CPPTRAJ: Software for processing and analysis of molecular dynamics trajectory data. Journal of Chemical Theory and Computation, 9(7):3084-3095, 2013. ISSN 15499618. doi: 10.1021/ct400341p. URL https://doi.org/10.1021/ct400341p. 6.3

[82] L Blum and Y Rosenfeld. Relation between the free energy and the direct correlation function in the mean spherical approximation. Journal of Statistical Physics, 63:1177-1190, 1991. 6.4

[83] W Nonner, L Catacuzzeno, and B Eisenberg. Binding and selectivity in L-type calcium channels: a mean spherical approximation. Biophysical Journal, 79(4):1976-1992, 2000. 6.4

[84] John Towns, Timothy Cockerill, Maytal Dahan, Ian Foster, Kelly Gaither, Andrew Grimshaw, Victor Hazlewood, Scott Lathrop, Dave Lifka, Gregory D Peterson, et al. Xsede: Accelerating scientific discovery computing in science \& engineering, 16 (5): 62-74, sep 2014. URL https://doi. org/10.1109/mcse, 2014. 7

[85] Xiaomin Wu, Gang Yang, and Lijun Zhou. The multi-factor coupled protein folding: Insights from molecular dynamics simulations. In Advances in Mathematical Chemistry and Applications, pages 265-299. Elsevier, 2015. S8.5

[86] Tingjun Hou, Junmei Wang, Youyong Li, and Wei Wang. Assessing the Performance of the MM/PBSA and MM/GBSA Methods. 1. The Accuracy of Binding Free Energy Calculations Based on Molecular Dynamics Simulations. Journal of chemical information and modeling, 51(1):69-82, 2011. S8.5

[87] Jianbo Hou, Zhiyang Zhang, and Louis A Madsen. Cation/Anion Associations in Ionic Liquids Modulated by Hydration and Ionic Medium. The Journal of Physical Chemistry B, 115(16):4576-4582, 2011. S8.5

[88] Tingjun Hou, Junmei Wang, Youyong Li, and Wei Wang. Assessing the performance of the molecular mechanics/Poisson Boltzmann surface area and molecular mechanics/generalized Born surface area methods. II. The accuracy of ranking poses generated from docking. Journal Of Computational Chemistry, $32(5): 866-877,2011$. S 8.5

[89] Stephen C Hoops, Kenneth W Anderson, and Kenneth M Merz Jr. Force field design for metalloproteins. Journal of the American Chemical Society, 113(22):8262-8270, 1991. S8.5 


\section{Acronyms}

$\alpha \mathbf{P V} \alpha$-parvalbumin. 1-20, 22, 23, 27-35

$\beta \mathbf{P V} \beta$-parvalbumin. $2,3,14,27$

$\mathbf{C a}^{2+}$ calcium. 1-9, 11-33, 35

CaM calmodulin. 16

CBP Ca-binding protein. 1, 2, 16

CBPs Ca-binding proteins. 3, 18

$\mathrm{Cl}^{-}$chloride. 6

$\mathbf{K}^{+}$potassium. 6

$\mathbf{M g}^{2+}$ magnesium. 1-7, 9, 11-26, 28-33

MM/GBSA molecular mechanics generalized Born approximation. 1, 3-7, 11, 13-16, 18-21

MSA mean spherical approximation. 1-3, 6, 19, 21, 27

PV Parvalbumin. 1, 2, 16, 19

RMSD root mean squared deviations. 3

RMSF root mean squared fluctuations. 3

TnC troponin C. 3, 15, 16, 19

WT wild-type. $3,5,6,11,13,14,16,33$ 